\title{
PTHrP drives breast tumor initiation, progression, and metastasis in mice and is a potential therapy target
}

\author{
Jiarong Li,, ${ }^{1}$ Andrew C. Karaplis, ${ }^{2}$ Dao C. Huang, ${ }^{1}$ Peter M. Siegel, ${ }^{3}$ Anne Camirand,1,2 \\ Xian Fang Yang, ${ }^{1}$ William J. Muller, ${ }^{3}$ and Richard Kremer ${ }^{1}$

\begin{abstract}
'Department of Medicine, McGill University Health Centre, Montréal, Quebec, Canada. ${ }^{2}$ Lady Davis Institute for Medical Research,
\end{abstract} \\ Jewish General Hospital, Montréal, Quebec, Canada. ${ }^{3}$ Goodman Cancer Research Centre, McGill University, Montréal, Quebec, Canada.
}

\begin{abstract}
Parathyroid hormone-related protein (PTHrP) is a secreted factor expressed in almost all normal fetal and adult tissues. It is involved in a wide range of developmental and physiological processes, including serum calcium regulation. PTHrP is also associated with the progression of skeletal metastases, and its dysregulated expression in advanced cancers causes malignancy-associated hypercalcemia. Although PTHrP is frequently expressed by breast tumors and other solid cancers, its effects on tumor progression are unclear. Here, we demonstrate in mice pleiotropic involvement of PTHrP in key steps of breast cancer - it influences the initiation and progression of primary tumors and metastases. Pthrp ablation in the mammary epithelium of the PyMT-MMTV breast cancer mouse model caused a delay in primary tumor initiation, inhibited tumor progression, and reduced metastasis to distal sites. Mechanistically, it reduced expression of molecular markers of cell proliferation (Ki67) and angiogenesis (factor VIII), antiapoptotic factor Bcl-2, cell-cycle progression regulator cyclin D1, and survival factor AKT1. PTHrP also influenced expression of the adhesion factor CXCR4, and coexpression of PTHrP and CXCR4 was crucial for metastatic spread. Importantly, PTHrP-specific neutralizing antibodies slowed the progression and metastasis of human breast cancer xenografts. Our data identify what we believe to be new functions for PTHrP in several key steps of breast cancer and suggest that PTHrP may constitute a novel target for therapeutic intervention.
\end{abstract}

\section{Introduction}

Metastases to bone, lung, and other organs are common and catastrophic consequences of breast cancer progression; most patients do not die from the primary tumor, but because of cancerous invasion to distal sites $(1,2)$. Once breast cancer metastases are established in bone or lung, the condition is generally considered incurable. There is therefore an urgent need to improve current therapies that address cancer spread, and an ideal solution will target upstream signaling molecules to prevent compensatory mechanisms that can result from blockade of individual downstream signaling points $(3,4)$.

Parathyroid hormone-related protein (PTHrP, also referred to as parathyroid hormone-like protein [PTHLP]) is a secreted factor expressed in almost all normal fetal and adult tissues. The 13 $\mathrm{N}$-terminal amino acids of PTHrP are highly homologous to those of parathyroid hormone (PTH), a characteristic that allows PTHrP to act through the type $1 \mathrm{PTH}$ receptor (PTH1R) (5). The rest of the PTHrP amino acid sequence is unique, however, and confers to the molecule many properties resulting from signal transduction cascades and nuclear translocation distinct from those of PTH (6). $\mathrm{PTHrP}$ acts as an autocrine, paracrine, or intracrine factor in a wide range of developmental and physiological processes $(7,8)$, it has growth-promoting and antiapoptotic properties (6), and it plays a crucial role in the development of the mammary gland and skeleton (8-10). Of special interest is the association of PTHrP with oncologic pathologies such as breast cancer $(11,12)$ and lung $(13-15)$, prostate (16-18), renal (19), colorectal $(20-22)$, skin $(23,24)$,

Conflict of interest: The authors have declared that no conflict of interest exists. Citation for this article: J Clin Invest. 2011;121(12):4655-4669. doi:10.1172/JCI46134. and gastric carcinomas $(25,26)$. Circulating levels of PTHrP generally correlate with the more advanced stages of cancer (20,27-32), and PTHrP regulates the expression of several tumor-relevant genes (33). Despite the frequent association of PTHrP dysregulation with many tumor types, a precise and direct role for PTHrP in cancer development and progression has been difficult to prove, and its involvement in tumor initiation in vivo and in critical steps of malignant conversion is not clear.

Here, we demonstrate PTHrP implication in key steps of breast cancer initiation, progression, and metastasis. We show that PTHrP plays a major role in stimulation of breast tumor growth rates and metastatic spread to distal organs through its effects on several crucial control molecules, including prosurvival signal molecule AKT and chemokine receptor CXCR4.

\section{Results}

Pthrp ablation occurring after birth allows normal mammary development. To clarify the role of PTHrP in tumorigenesis, the human breast cancer mouse model PyMT-MMTV (where the $\mathrm{mT}$ oncogene drives oncogenic transformation; ref. 34) was used to generate animals with a Cre-loxP-mediated (35) hetero- or homozygous Pthrp gene ablation specifically targeted to the mammary epithelium (ME) (Supplemental Figure 1, A and B; supplemental material available online with this article; doi:10.1172/JCI46134DS1). All animals used in the present study were confirmed by marker analysis to possess more than 99\% FVB/NJ background. In standard PyMT-MMTV animals, tumors appeared spontaneously, approximately $100 \%$ of these tumors expressed PTHrP (55 tumors tested by RT-PCR), and their PTHrP expression increased with age (Figure 1A). In contrast, in Pthrp-ablated animals, spontaneous breast tumors showed 
A
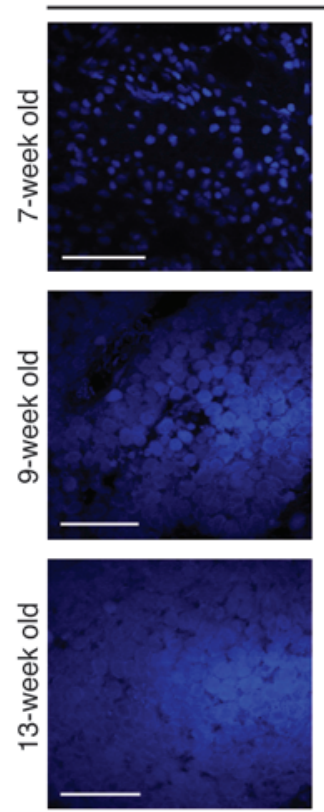

DAPI
PyMT mice
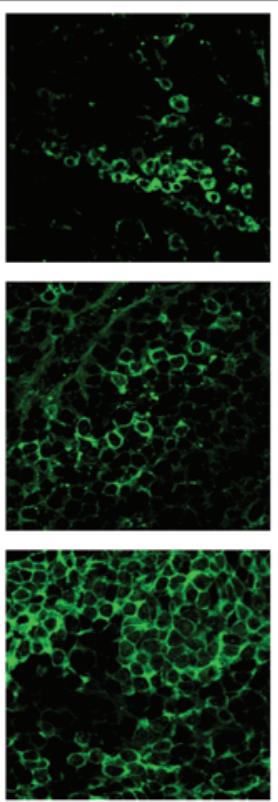

PTHrP

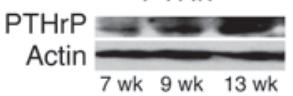

C Pthrp ${ }^{w T} ;$ Cre

control
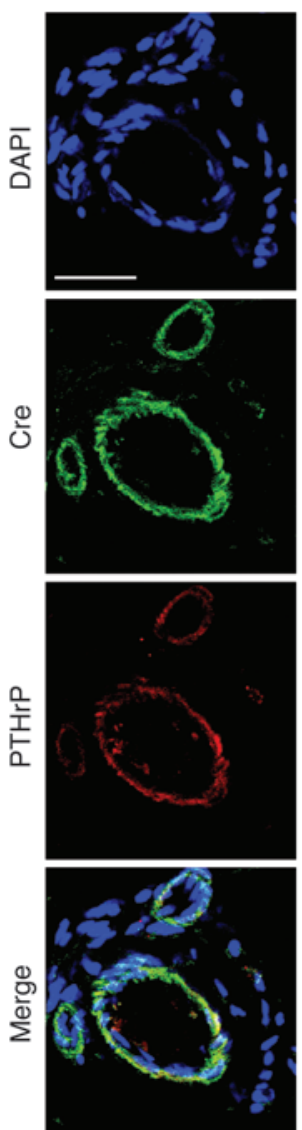

Pthrp floxhlox $; \mathrm{Cre}^{+}$

homozygous
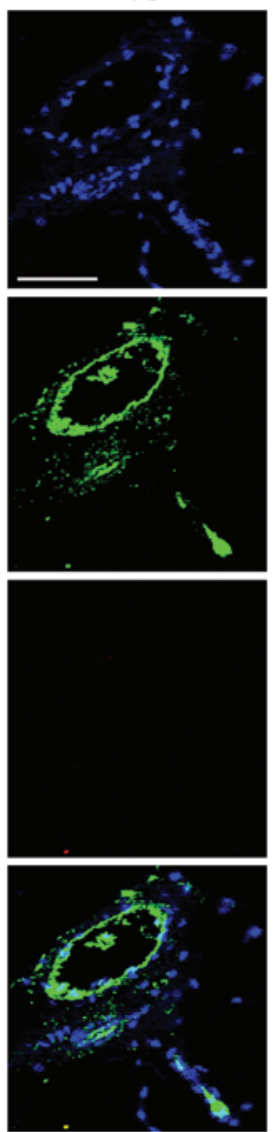

B
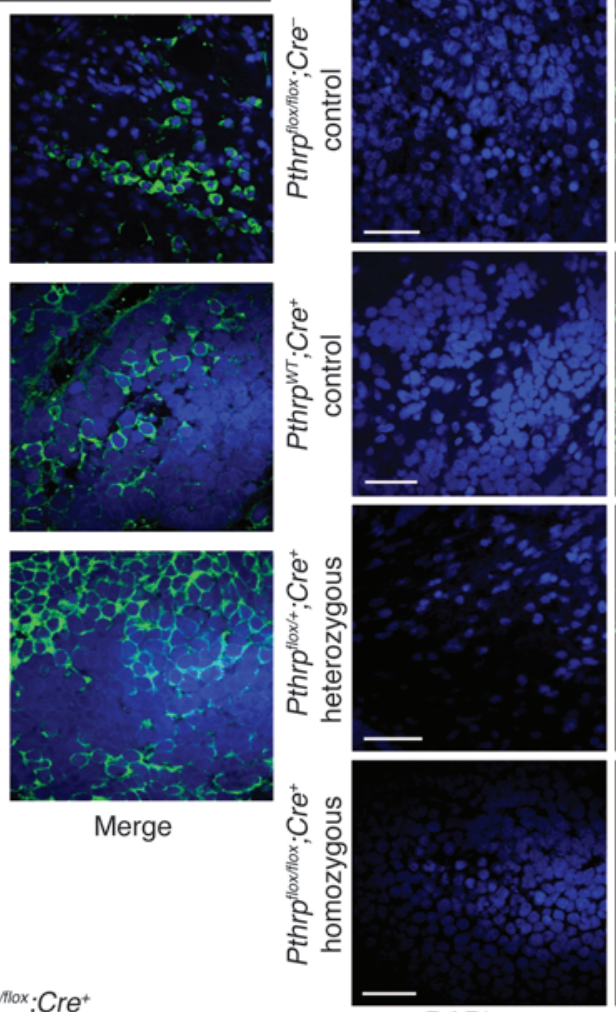

DAPI

PTHrP

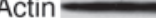

20.0

\section{D}
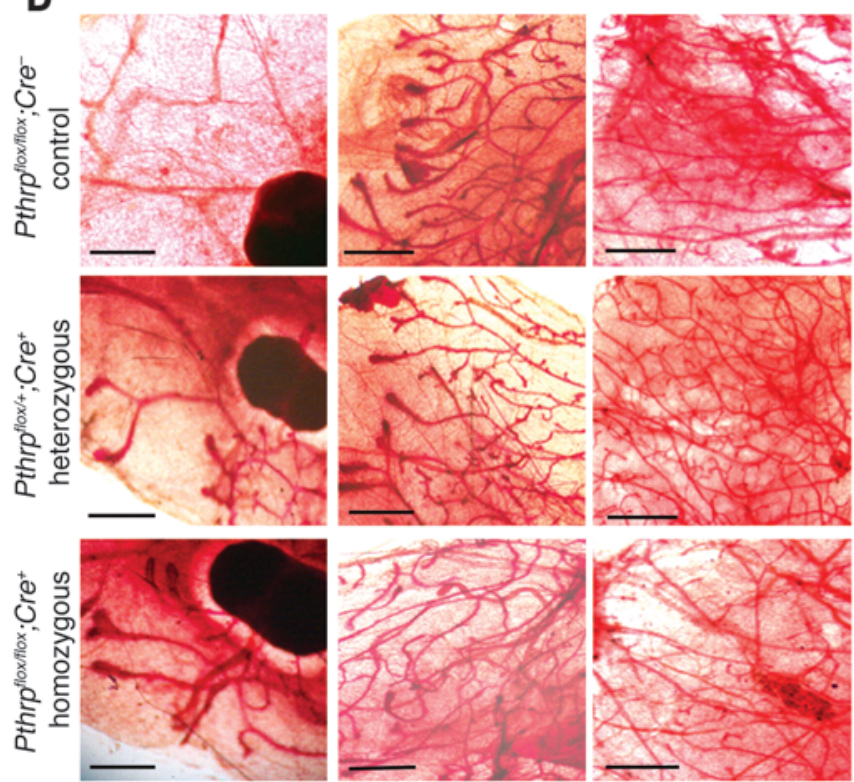

3-week old

E

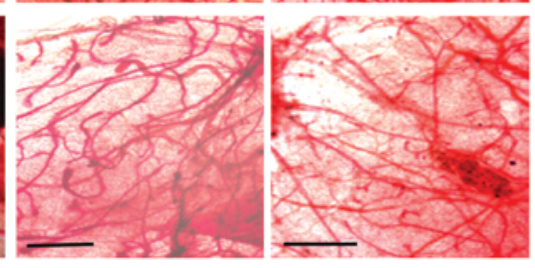

5-week old

7-week old

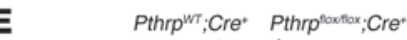

PTHrP control homozygous

Actin 


\begin{abstract}
Figure 1
Cre-mediated Pthrp ablation in ME allows normal mammary development. (A) Confocal images of IF staining with anti-PTHrP antibody in spontaneous breast tumors from standard PyMT mice and Western blot quantification showing increasing PTHrP expression in these tumors with respect to age. (B) Confocal images of IF staining for DAPI (blue) and PTHrP (green) in tumor tissues from control

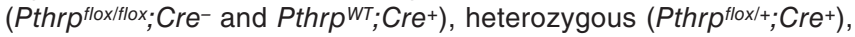
and homozygous (Pthrp ${ }^{\text {flox fllox }}$;Cre ${ }^{+}$) transgenic animals showing incremental decrease in PTHrP expression with allele ablation. Lower panel: Western blot quantification for PTHrP expression in tumors from the various genotypes. (C) Confocal images of IF staining with DAPI (blue), Cre recombinase (green), and PTHrP (red) showing colocalization of Cre and PTHrP. (D) Whole-mount staining analysis (Neutral Red) of mammary glands showing ductal outgrowth at 3, 5, and 7 weeks for control, heterozygous, and homozygous female virgin mice. (E) Western blot of PTHrP expression in mammary glands of virgin 7-week-old mice. Scale bars: $50 \mu \mathrm{m}(\mathbf{A}-\mathbf{C})$; $5 \mu \mathrm{m}$ (D).
\end{abstract}

incremental reduction in PTHrP expression from Pthrpflox/flox; $\mathrm{Cre}^{-}$(control) to $\mathrm{Pthrp}^{\text {flox/+}} ; \mathrm{Cre}^{+}$(heterozygous) to Pthrpflox/flox; $\mathrm{Cre}^{+}$ (homozygous) (Figure 1B). Pthrp $p^{W T / W T}$; Cre $e^{+}$animals were generated to test potential artifactual side effects caused by expression of Cre recombinase, but showed no difference from other controls throughout all experiments.

When tumor cells were cultured in vitro, radioimmunometric assays for PTHrP in the conditioned medium indicated low residual expression of PTHrP in homozygous ablated cells as follows: $\mathrm{Cr}^{-}$control cells, $178.7 \pm 33.6 \mathrm{pg} / \mathrm{ml}$; $\mathrm{Cre}^{+}$tumor-derived cells, $10.1 \pm 2.3 \mathrm{pg} / \mathrm{ml}$; mean $\pm \mathrm{SD}, n=13$ and 10 mice, respectively. In tumor-bearing mice, circulating PTHrP was undetectable, and calcium serum concentrations were not significantly different between control $(2.28 \pm 0.39 \mathrm{mmol} / \mathrm{l})$ and Pthrp-ablated mice $(2.23 \pm 0.23 \mathrm{mmol} / \mathrm{l})$.

PTHrP expression in the normal gland was localized both in luminal epithelial cells and in myoepithelial cells (Supplemental Figure 2, A-C, and ref. 36). PTH1R expression was unaffected by Pthrp ablation (Supplemental Figure 3).

Immunofluorescence (IF) staining confirmed Cre expression in the luminal epithelium of control mice (Figure 1C). The Cre gene was expressed under the control of the MMTV-long terminal repeat (MMTV-LTR), and its expression was detectable from 6 days postpartum (37). Pthrp ablation therefore occurred after birth, and mammary glands from 3-, 5-, and 7-week-old virgin females presented normal ductal outgrowth as well as normal lactation capacity with no detectable differences among genotypes (Figure 1D). PTHrP in the mammary gland was substantially reduced in 7-week-old homozygous mice in comparison with control animals (Figure 1E). These data show that ME-targeted Pthrp deletion can be achieved without hindering normal mammary gland development.

Pthrp ablation delays breast cancer initiation and primary tumor progression. Tumor hyperplasia was detectable in the mammary gland of control animals as early as 27 days after birth, adenomas were visible by 35 days, and early adenocarcinomas were visible by day 45 (Figure 2A). In contrast, ablation of both Pthrp alleles significantly delayed tumor initiation (Figure 2A). Tumor growth over time was reduced in homozygous Pthrpflox/flox; $\mathrm{Cre}^{+}$animals in comparison with Pthrpt flox/flox; $\mathrm{Cre}^{-}$and Pthrp ${ }^{W T}$; $\mathrm{Cre}^{+}$controls (Figure 2B), and tumor weight/mouse at sacrifice (13 weeks) was $70 \%$ lower in ablated animals than in controls (Figure 2C). While $100 \%$ of control mice presented palpable tumors (2-mm diameter) around day 55, heterozygous and homozygous animals reached this percentage by days 75 and 92, respectively (Figure 2D). Homozygous mice reached tumor size requiring humane sacrifice at a much later age than control and heterozygous mice (Figure 2E). Tumors from hetero- and homozygous mice were 50\% and $75 \%$ smaller than their respective $\mathrm{Cr}^{-}$controls, and there were $35 \%$ and $60 \%$ fewer tumors in heterozygous and homozygous mice at 13 weeks (Figure 3, A and B, and Supplemental Table 1). The expression of Cre was not involved in the changes observed in tumor delay, as demonstrated by the Pthrp ${ }^{W T}$; $C r e^{+}$controls, which did not differ from the other controls (Figure 2, B-E, and Figure 3, A and B). These results show that ablation of the Pthrp gene in the ME of PyMT-MMTV mice significantly delays primary breast cancer initiation events, even before tumors reach a palpable stage, and reduces their subsequent growth.

More complete Pthrp ablation by Cre-carrying adenovirus further delays breast cancer initiation and progression. Because some residual PTHrP expression was detectable in approximately $20 \%$ of primary tumors from Pthrfflox/flox; $\mathrm{Cre}^{+}$animals (Figure 1B), a more complete Pthrp knockout was achieved by transfecting isolated cells from $P$ thr $p^{f l o x} / f l o x$; $\mathrm{Cre}^{-}, \mathrm{Pthrp}^{f l o x /+} ; \mathrm{Cr} e^{-}$and $\mathrm{Pthrp} \mathrm{WT}^{\mathrm{WT}}$ tumors with an adenovirus containing a CreGFP sequence or with a control adenovirus containing GFP only. Transfected adenoGFP control or adenoCre ${ }^{+}$cells purified by cell sorting were transplanted into the fourth mammary fat pad (MFP) of healthy FVB syngeneic mice $\left(5 \times 10^{5}\right.$ cells). PTHrP expression was eliminated in the adenoCre ${ }^{+}$-derived tumors (Figure 4, A and B), tumor volume was greatly decreased (Figure 4C), and tumor load at injection site was dramatically reduced (Figure 4, $\mathrm{D}$ and $\mathrm{E})$. These results show that more stringent Pthrp ablation conditions enhance tumor inhibition.

Pthrp ablation modifies cell-cycle, apoptosis, and angiogenesis events. Decreasing PTHrP expression reduced Ki67 (cell proliferation), factor VIII (angiogenesis), and cyclin D1 staining (Figure 5, A and B). Propidium iodide flow cytometry analysis revealed that $53.7 \% \pm 1.19 \%$ of control Pthrptox/flox; $\mathrm{Cre}^{-}$remained in $\mathrm{G}_{0} / \mathrm{G}_{1}$ compared with $71.32 \% \pm 3.70 \%$ of homozygous Pthrfflox/flox; $\mathrm{Cre}^{+}$. In contrast, $18.34 \% \pm 1.80 \%$ of control Pthrpflox/flox; Cre C $^{-}$and $8.10 \% \pm 4.00 \%$ of homozygous Pthrfflox/flox; $\mathrm{Cre}^{+}$tumor cells were in $\mathrm{S}$ phase, an observation consistent with the cyclin D1 decrease (not shown). Importantly, in cultured cells isolated from tumors, cyclin D1 expression colocalized specifically with cells that escaped PTHrP ablation (Figure 5C), suggesting that in this system, PTHrP is crucial for cyclin D1 expression. Pthrp ablation was accompanied by an increase in TUNEL apoptotic staining in tumors and tumorderived cultured cells (Figure 5D) and by a decrease in Bcl-2 expression (Figure 5B). Western blots showed no difference in expression levels for factor VIII, cyclin D1, and Bcl-2 between Pthrfflox/flox; Cre ${ }^{-}$ and $P$ thrp ${ }^{W T}$; $C r e^{+}$controls (Supplemental Figure 4), indicating that decreases in signaling molecule levels can be attributed to Pthrp ablation. Overall, these data indicate a pleiotropic effect of PTHrP in cell-cycle, apoptosis, and angiogenesis events.

PTHrP is involved in CXCR4 and AKT expression control. Pthrp ablation was accompanied by inhibition of metastasis marker CXCR4 expression in primary breast tumors of the same size (controls: 13 weeks; homozygous animals: 18 weeks; Figure 6, A and B). Furthermore, CXCR4 in cells isolated from primary tumors appeared exclusively in cells that avoided Pthrp ablation (Figure 6B). Pthrp ablation also decreased AKT1 total protein and increased total AKT2 in tumor tissues (Figure 6, C and D), and 
A
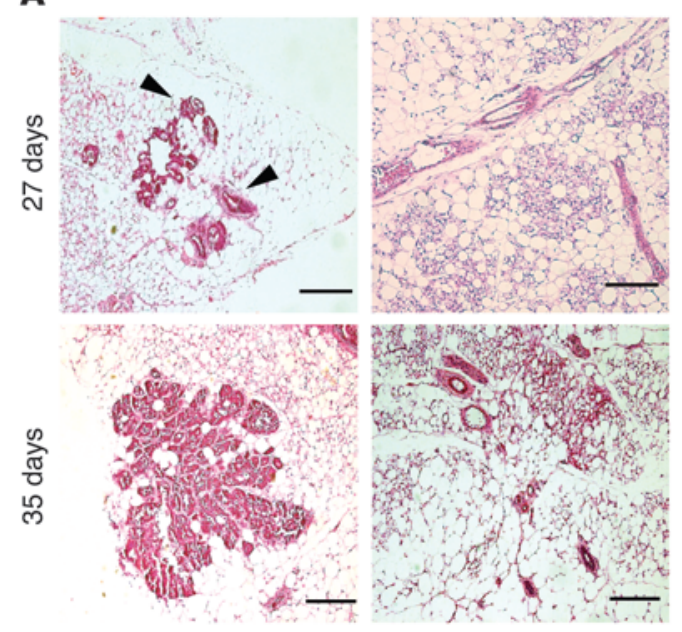

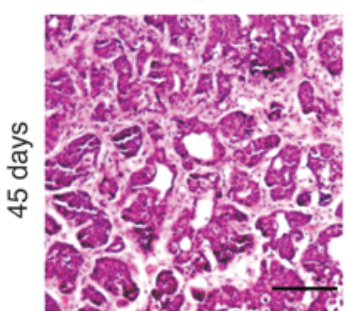

Pthrp floxilox $; C^{-}$

control

D

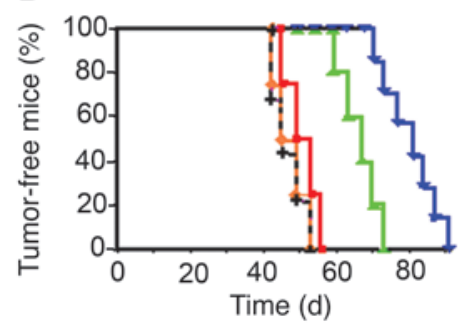

B

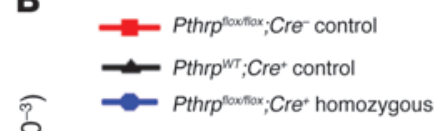

0.4
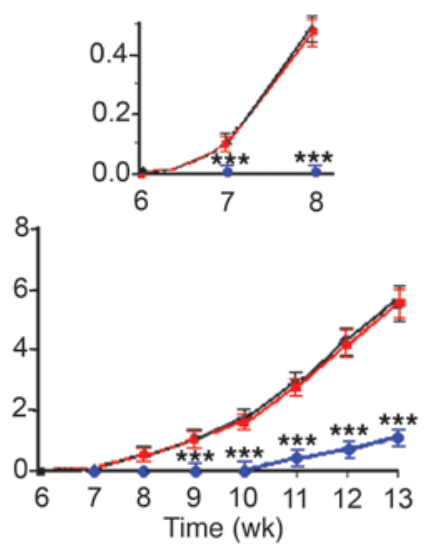

C

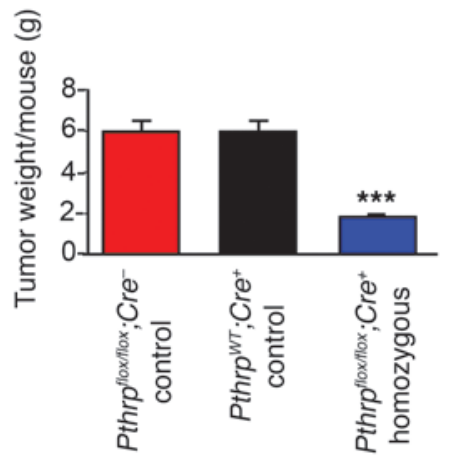

E

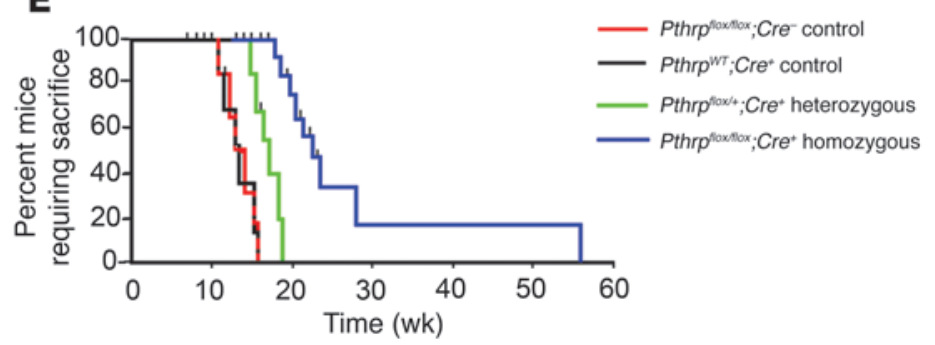

Figure 2

Pthrp ablation delays breast cancer initiation and progression. (A) H\&E staining of breast tissues from control (Pthrp flox/flox; Cre $\left.{ }^{-}\right)$and homozygous $\left(\right.$ Pthrp flox/flox; $\left.\mathrm{Cre}^{+}\right)$animals at 27, 35, and 45 days. Hyperplasias (arrowheads) are not detectable in homozygous animals before 45 days. Scale bars: $200 \mu \mathrm{m}$. (B) Tumor volume over time for control (Pthrp flox/flox;Cre- and Pthrp ${ }^{\text {WT; }}$; Cre $\left.{ }^{+}\right)$and homozygous mice $($Pthrp flox/flox;Cre+ $)$showing that the delay in tumor growth is not related to the expression of the Cre gene. Inset: early time points. Values shown represent mean $\pm \mathrm{SD}, n=12$ mice per group. ${ }^{\star \star *} P<0.001$. (C) Tumor weight per mouse at sacrifice (13 weeks) for control and homozygous animals. Values shown represent mean $\pm \mathrm{SD}, n=12$ mice per group. ${ }^{\star \star *} P<0.001$. (D) Kaplan-Meier analysis of tumor onset for mice of all genotypes illustrating allelic effect for Pthrp ablation. (E) Kaplan-Meier analysis showing that control mice reach age requiring sacrifice much earlier than homozygous animals.

most residual AKT1 colocated with residual PTHrP in $\mathrm{Cr}^{+}$cultured cells isolated from tumors (Figure 6E). Again, the expression of Cre was not involved in changes seen for AKT or CXCR4 (Supplemental Figure 4). Phosphorylation of AKT1 (Ser473) was also inhibited by Pthrp ablation (Figure 6F). siRNA experiments targeting AKT1 effectively decreased its levels in control $\left(\mathrm{Cre}^{-}\right)$and Pthrp-ablated (Cre ${ }^{+}$) tumor cells (Figure 7A). Cell proliferation was reduced by $38.9 \% \pm 8.0 \%$ by Pthrp ablation alone (Figure $7 \mathrm{~B}$ ), by $46.7 \% \pm 4.1 \%$ through AKT1 knockdown alone, and by $80.2 \% \pm 2.2 \%$ in Pthrp-ablated cells combined with AKT1 siRNA. These data indicate that PTHrP is involved in the control of expression of survival molecules AKT1 and AKT2 and of the chemokine receptor CXCR4.
PTHrP drives metastatic spread to peripheral blood, bone marrow, and lungs, and distal metastases comprise PTHrP/CXCR4 double-positive cells. Cells from control tumors possessed more than twice the Matrigel invasiveness potential of cells from Pthrp-ablated mice (Figure 8A), and their motility after surface wounding was higher (Figure $8 \mathrm{~B}$ ). There were fewer circulating tumor cells in homozygous than in control animals (same-size tumors, controls: 13 weeks; homozygous: 18 weeks; Figure 8C). Tumor cells flushed from bone marrow were detectable only in control animals (average of $31.5 \pm 3.4$ cells per animal, $n=4$; Figure $8 \mathrm{D}$ ).

Similarly, the incremental decrease in PTHrP lowered the number of micro- and macroscopic metastatic lesions in lung tissues. At 13 weeks, $100 \%$ (45/45) of control mice presented lung metastases compared 
A

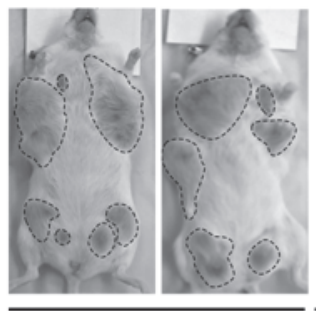

Pthrp floxfliox;Crecontrol

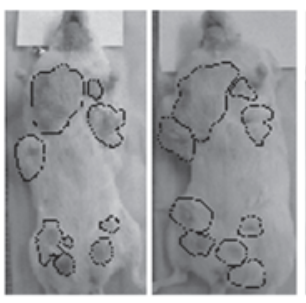

Pthrp ${ }^{W T} ;$ Cre $^{+}$ control

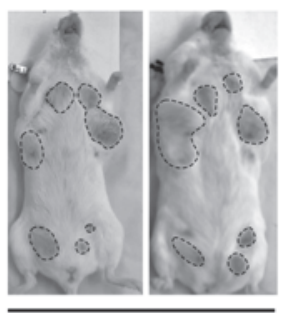

Pthrp ${ }^{f l o x /+} ;$ Cre $^{+}$ heterozygous

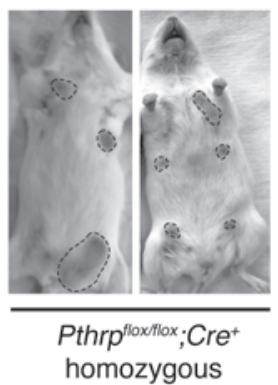

homozygous
B

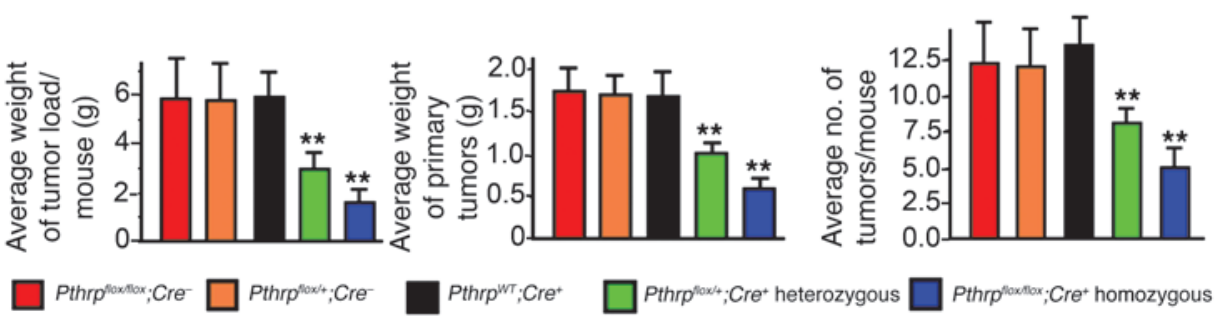

\section{Figure 3}

Pthrp ablation reduces spontaneous tumor load. (A) Tumor load in whole animals (13 weeks). (B) Average weight of tumor load per mouse, average weight of primary tumors, and average number of tumors per mouse at sacrifice for the same genotypes. Values shown represent mean \pm SD; $n=20$ for Pthrp flox/flox; Cre $^{-}, n=18$ for Pthrp $^{\text {flox } /+} ;$ Cre $^{-}, n=22$ for Pthrp WT/WT; Cre ${ }^{+}$(controls), $n=12$ for Pthrp ${ }^{\text {flox/+;}} \mathrm{Cre}^{+}$(heterozygous), and $n=30$ for Pthrp flox/flox; $\mathrm{Cre}^{+}$ (homozygous), ${ }^{\star *} P<0.01$. with $47 \%$ (8/17) for heterozygous and $0 \%$ for homozygous animals (0/18) (Figure 8E). At 18 weeks, when same-size tumors were achieved in homozygous mice, smaller lung tumors appeared in $40 \%$ $(12 / 30)$ of homozygous mice compared with $100 \%$ of control mice euthanized at 13 weeks for humane reasons (Figure 8, F and G). When tumor cells were transfected with adenoCreGFP or adenoGFP virus, purified, and implanted $\left(5 \times 10^{5}\right.$ cells $)$ in the fourth MFP of syngeneic mice, same-size primary tumors in the MFP were achieved at 8 weeks in control, 11 weeks in heterozygous, and 16 weeks in homozygous mice (not shown). At those stages, lung metastases were present in $70 \%(16 / 23)$ of control, 55\% (6/11) of heterozygous, and $0 \%(0 / 22)$ of homozygous mice (Figure $8, \mathrm{H}$ and I).

Importantly, in the spontaneous PyMT tumor model, lung metastases cells in control and homozygous mice (same-size tumors, 13 weeks and 18 weeks, respectively) were almost uniformly PTHrP and CXCR4 positive (Figure 9), indicating that lung metastases were mainly derived from PTHrP-expressing tumor cells that escaped gene ablation (Supplemental Figure 5). These results point to a driving role for PTHrP in invasion and metastasis in this system and suggest that combined expression of PTHrP and CXCR4 is crucial to metastatic spread.

Anti-PTHrP neutralizing $m A$ bs inhibit breast cancer progression in vitro and in vivo. $\mathrm{mAbs}$ were produced against human $\mathrm{PTHrP}_{1-33}$, and 2 molecules positive exclusively for this peptide (158 IgG, M45 IgM) significantly inhibited Matrigel invasion by human MDA-MB-435 breast cancer cells (PTHrP positive, secreting $300 \pm 30 \mathrm{pg} / \mathrm{ml}$ of PTHrP in the conditioned medium) compared with cells treated with a control antibody (Figure 10A). MDA-MB-435 cells were also injected into the MFPs of BALB/c nu/nu mice and animals treated with the mAbs $(200 \mu \mathrm{g}$ subcutaneously every 48 hours for 6 weeks). Primary tumor growth in untreated animals reached $1.5-2.0 \mathrm{~cm}^{3}$ by 6 weeks after tumor transplantation. A significant inhibition $(P<0.01)$ in the size of primary tumor growth was observed in mice treated with 158 or M45 compared with controls (Figure 10B). The 158 antibody reduced the expression of CXCR4 and AKT1 in mammary tumors developing from injected cells (Figure 10C). After 6 weeks, approximately 100\% of control animals presented lung metastases in contrast with $33 \%$ for mAb-treated mice (Figure 10, D and E), and metastases from lungs of antibody-treated mice were fewer and 50\% smaller than those from vehicle-treated controls (Figure 10, F and G). CXCR4 expression was still prevalent in lung metastases despite antibody treatment (Figure 10H), suggesting that either efficacy of PTHrP neutralization was not optimized or that CXCR4 expression can be driven by additional factors. Liver and kidney tissues from animals treated with antibodies presented no histological evidence for toxicity due to treatment (not shown). These results suggest that anti-PTHrP antibodies can be considered for therapeutic use against human breast cancer progression.

Overall, our results indicate that PTHrP favors tumor cell survival in the primary site and plays a role in breast cancer progression through its control of cyclin D1, Ki67, Bcl-2, factor VIII, AKT1, AKT2, and CXCR4 levels (Figure 11). When tumor cells migrate to distal sites, adhesion to local ligands is facilitated by PTHrP's maintenance of CXCR4 levels, and its positive action on tumor proliferation allows consequent metastatic expansion of PTHrP/ CXCR4 double-positive cells.

\section{Discussion}

To investigate the involvement of $\mathrm{PTHrP}$ in breast cancer initiation, progression, and metastasis, we constructed an ME-specific Pthrpknockout animal in the PyMT-MMTV transgenic mouse model of breast tumorigenesis, using mice homozygous for a floxed Pthrp allele (2 loxP sites flanking exon 4, which encodes most of the PTHrP protein) (38). The PyMT mouse is a model with complete penetrance and approximates the 4 identifiable stages of tumor progression observed in human breast tumors (hyperplasia, adenoma, early carcinoma, and late carcinoma). These stages are followed in PyMT animals by a high frequency of distal metastasis, and the morphological characteristics and expression of biomarkers parallel those in the human cancer process (39). We obtained mice presenting various degrees of ablation of the Pthrp gene, demonstrating that reduction of PTHrP expression in ME brings substantial delays in breast cancer initiation without affecting mammary development. In Pthrp-ablated animals, primary tumor hyperplasia was considerably delayed, and palpable tumors appeared much later and were smaller and fewer than in control mice. Our observations are in agreement with several clinical studies in which patients with tumors produc- 
A
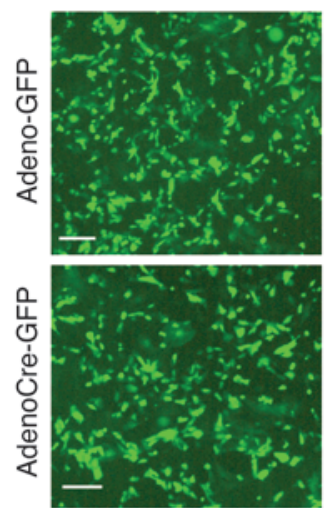

C

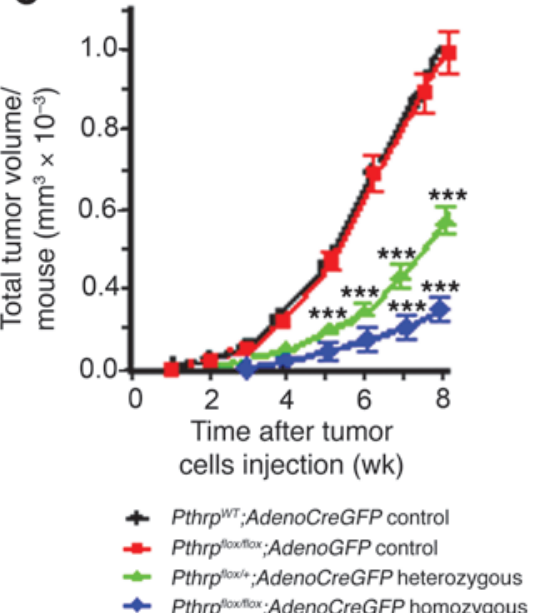

Tumors from injected adenovirus-transfected cells
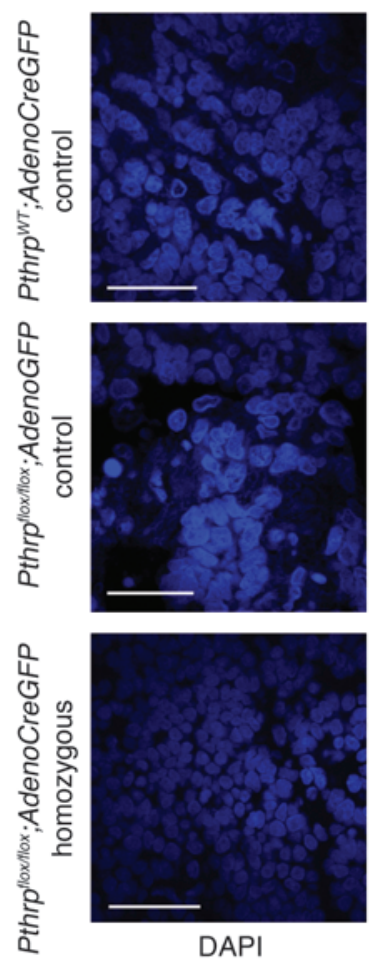

D

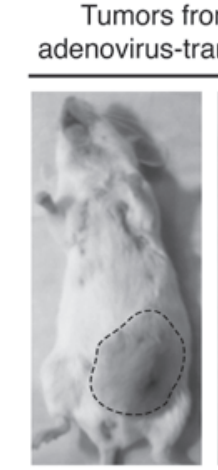

Pthrp floxhlox;

AdenoGFP control
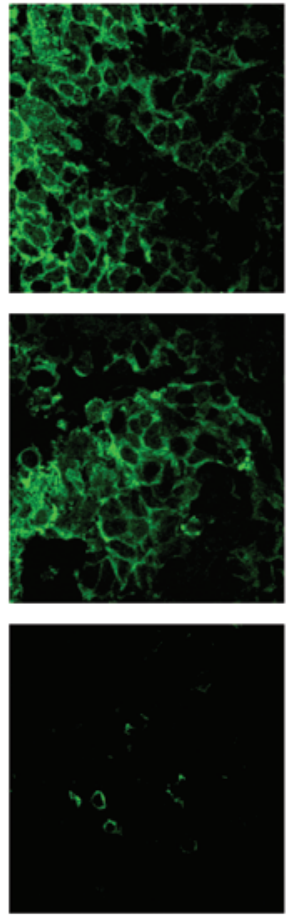

PTHrP

jected ransfected cells

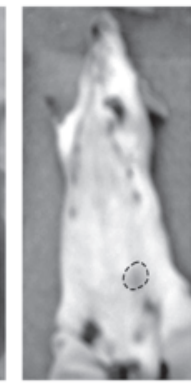

Pthrp AdenoCreGFP homozygous
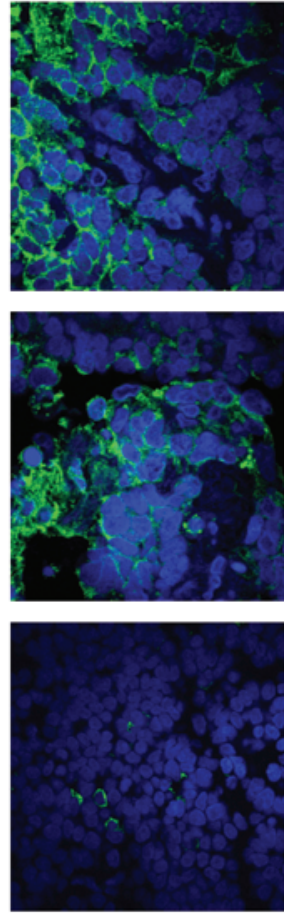

Merge
B

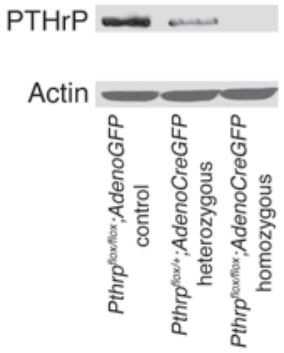

\section{Figure 4}

A more complete ablation of Pthrp by Cre-carrying adenovirus further delays breast cancer initiation and progression. (A) Adenovirus-transfected tumor cells selected by flow cytometry: left, cell fluorescence for GFP; right, confocal images of IF staining for DAPI (blue) and PTHrP (green) in mammary tumors derived from injected adenovirus-transfected tumor cells illustrating near-complete disappearance of PTHrP expression in homozygous tumors. Scale bars: $200 \mu \mathrm{m}$. (B) Western blot quantifying PTHrP expression in Pthrp floxfflox tumor cells transfected with adenoGFP. Lane 1, control, Pthrpflox/flox adenoGFP; lanes 2 and 3, hetero- and homozygous, Pthrp flox/+ or Pthrp flox/flox adenoCreGFP, respectively. (C) Tumor volume per animal for tumors derived from adenovirus-transfected tumor cells injected into the MFPs of syngeneic mice. Values represent mean \pm SD, $n=12$ mice for each group. ${ }^{* * \star} P<0.001$. (D) Tumor load in whole animals 8 weeks after adenovirus-transfected cell injection. (E) Average weight of breast tumor load per mouse at sacrifice. Values represent mean $\pm \mathrm{SD}, n=12$ mice per group. ${ }^{* \star} P<0.01$.

ing high PTHrP levels presented higher rates of metastasis and increased or earlier mortality (40-43). A recent report reached opposite conclusions, but relied on animal models with a very late-onset oncogenic system (neu based), which is more relevant to tumors aris- ing in older animals (44), and used mice with a heterogeneous genetic background. The age at which oncogenic induction occurs and the host's genetic background are 2 factors that significantly affect the biological behavior of tumors $(45,46)$ and likely account for the 
A
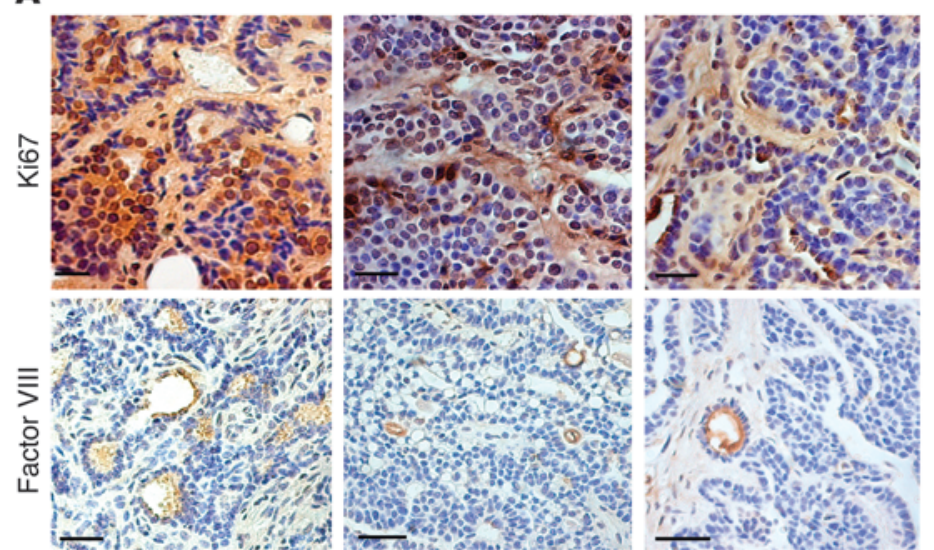

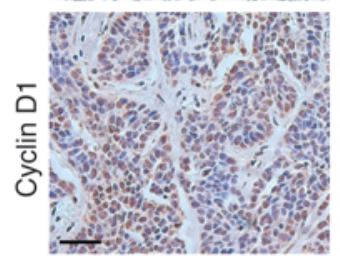

Pthrp floxflox; $\mathrm{Cre}^{-}$

control

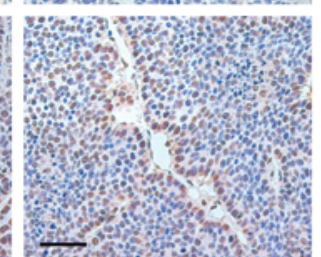

Pthrp ${ }^{\text {flox+ }} ; \mathrm{Cre}^{+}$

heterozygous

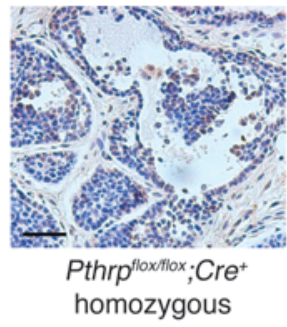

C
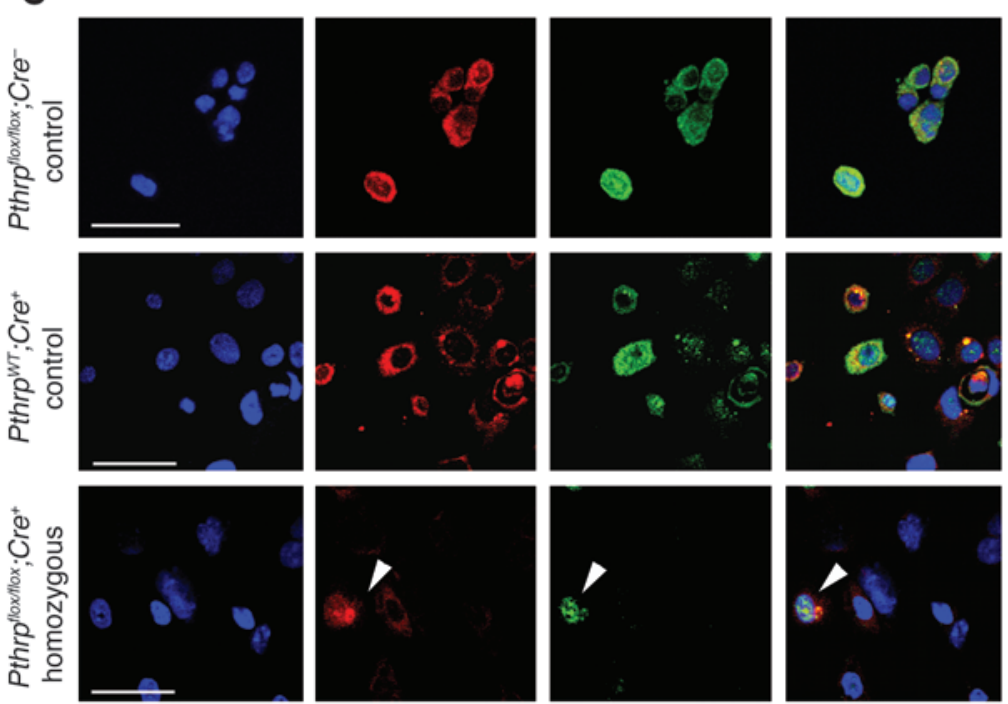

DAPI

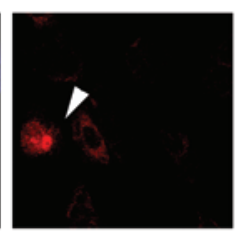

PTHrP

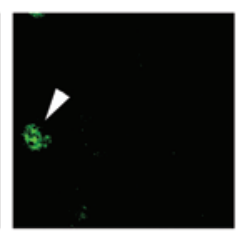

Cyclin D1

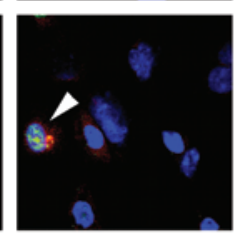

Merge

B

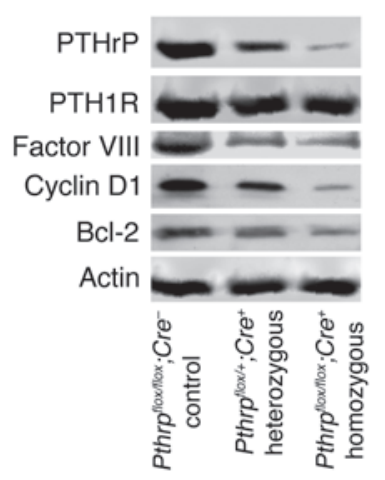

D

TUNEL

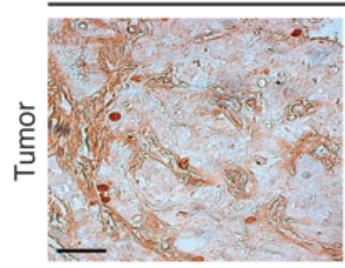

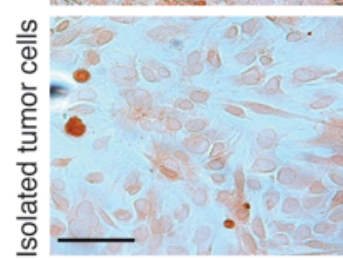

Pthrppoxtlox;Crecontrol
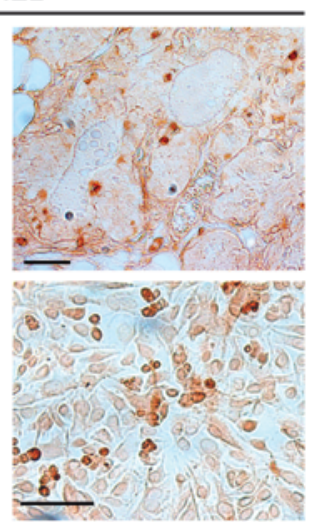

Pthrp ${ }^{\text {floxflox }} ; \mathrm{Cre}^{+}$ homozygous

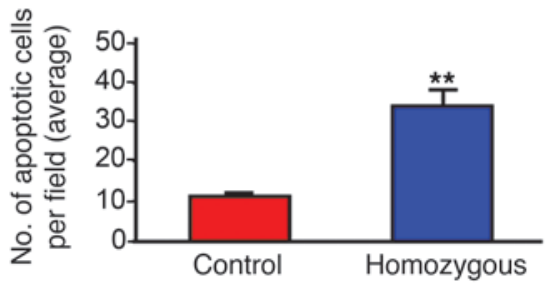

Figure 5

Pthrp ablation modifies cell-cycle, apoptosis, and angiogenesis events. (A) IHC staining of tumor tissues at 13 weeks showing a decrease in differentiation factor Ki67 (top), angiogenesis factor VIII (middle), and cyclin D1 (bottom) with degree of Pthrp ablation. (B) Western blot illustrating no change in PTH/PTHrP receptor 1 expression, but decreases in factor VIII, cyclin D1, and Bcl-2 with degree of Pthrp ablation. (C) Confocal images of IF staining in cultured cells isolated from tumors showing colocalization of PTHrP and cyclin D1 expression. The residual cells that escaped ablation and are still capable of expressing PTHrP are the only ones expressing cyclin D1 (homozygous, bottom row, arrowheads). Shown are DAPI (blue), PTHrP (red), and cyclin D1 (green). (D) TUNEL staining of breast tumor tissue (top) or in cells isolated from tumors and cultured (bottom), showing more abundant apoptotic events in homozygous tumors. Bottom panel: histogram showing average number of apoptotic cells per field in isolated tumor cells. Scale bars: $50 \mu \mathrm{m}$. Values represent mean $\pm \mathrm{SD}$. ${ }^{\star *} P<0.01$.

discrepancy observed with our results. Clinical studies reporting a good prognostic value for PTHrP in breast cancer have concentrated on cohorts in which neo-adjuvant cases were eliminated, therefore focusing on the less aggressive cases (47). In contrast, our animal model is more relevant to early-onset, pregnancy-independent, high- ly aggressive breast tumorigenesis, corresponding to a category of patients likely to require neo-adjuvant treatment.

In our mouse model, Pthrp ablation was accompanied by a nearcomplete disappearance of the CXCR4 receptor, a member of the chemokine superfamily that regulates cell migration and target- 
A

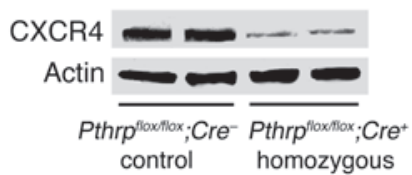

C
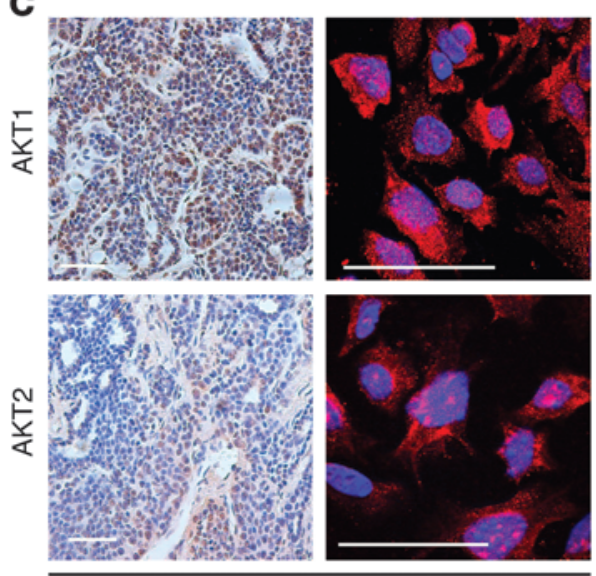

Pthrp ${ }^{\text {I0x/llox}} ;$ Cre $^{-}$control
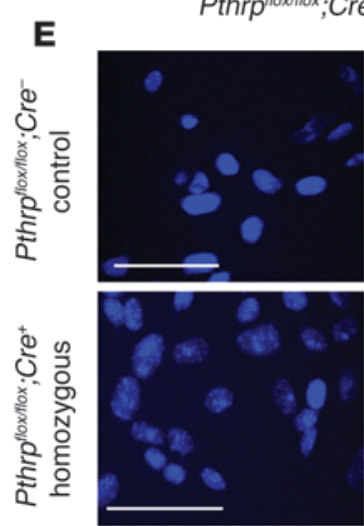

DAPI
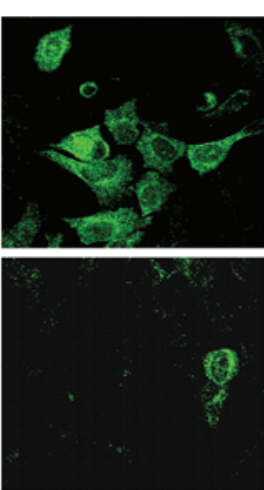

PTHrP

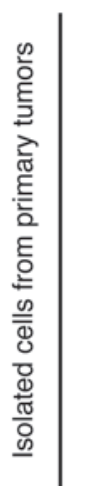

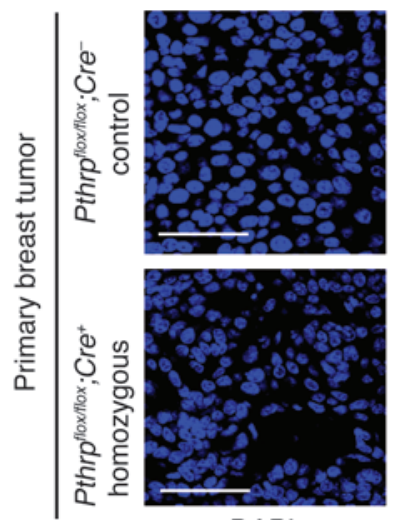

DAPI
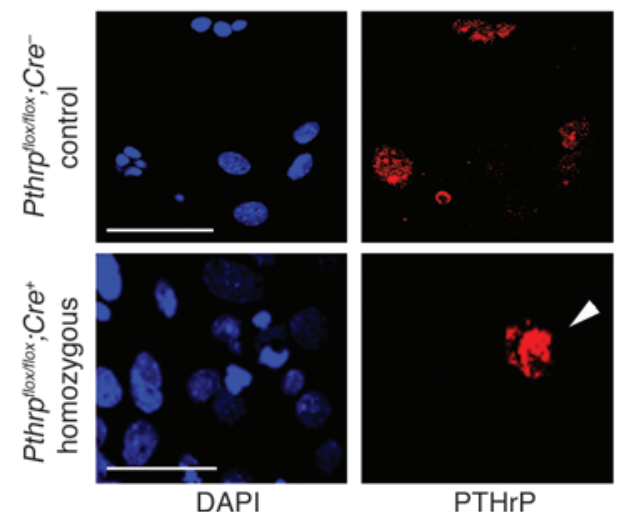

PTHrP
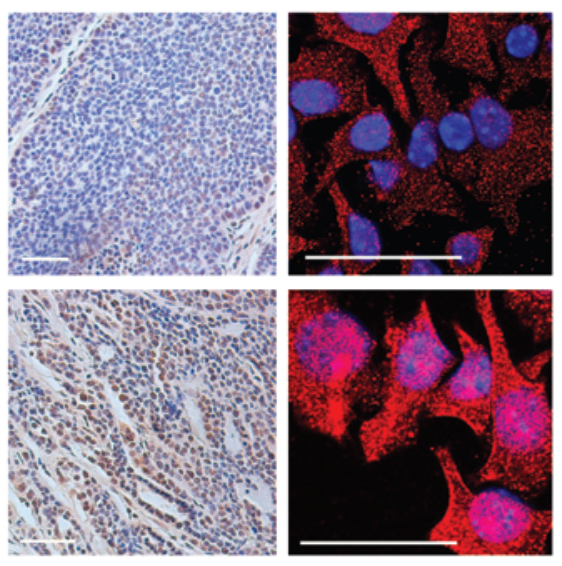

Pthrp $p^{\text {floxflox }} ; \mathrm{Cre}^{+}$homozygous
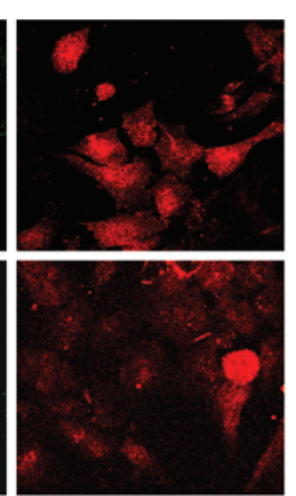

AKT1
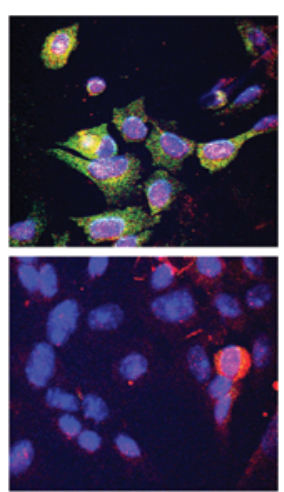

Merge

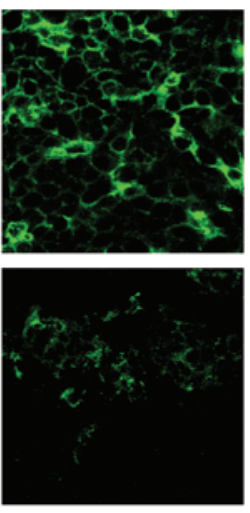

CXCR4
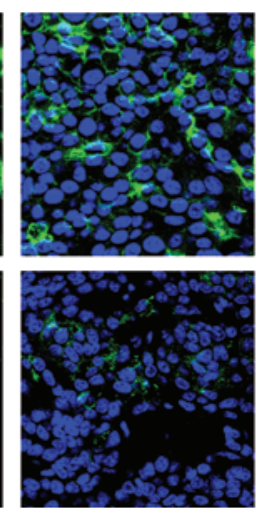

Merge
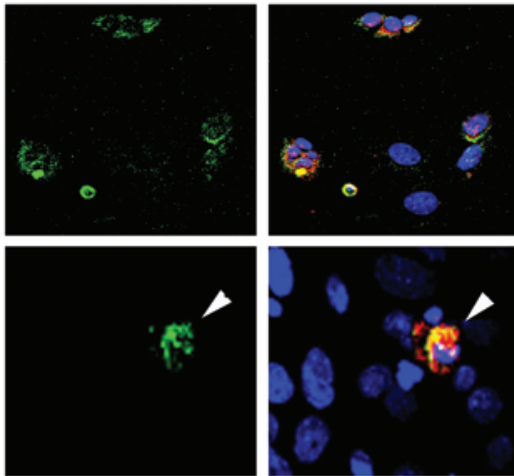

CXCR4

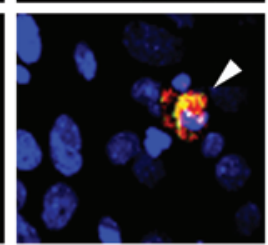

Merge

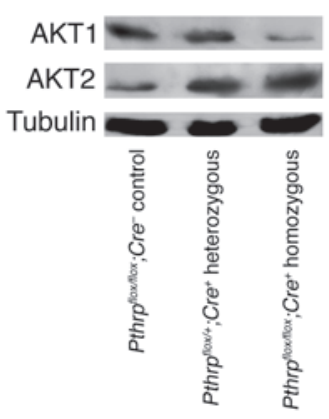

$\mathbf{F}$

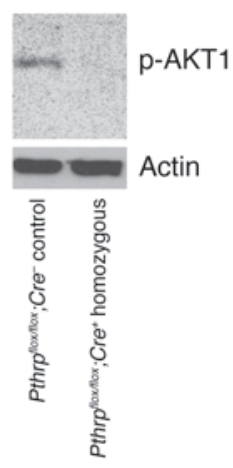




\section{Figure 6}

PTHrP is involved in CXCR4 and AKT expression control. (A) Western blot showing decreased CXCR4 expression in homozygous tumors. (B) Confocal images of IF. Top: primary breast tumors (control 13 weeks, homozygous mice 18 weeks). Bottom: cells isolated from these tumors and cultured. CXCR4 expression is significantly reduced with Pthrp ablation. Residual cells that escaped Pthrp ablation and are still expressing PTHrP are the only ones expressing CXCR4 (arrowheads). (C) IHC (left) and IF (right) images for AKT1 and AKT2 in tumors from control and ablated mice. Shown are DAPI (blue), AKT1 (top, red), and AKT2 (bottom, red). (D) Western blot showing decrease in AKT1 and increase in AKT2 concurrent with Pthrp ablation. (E) Confocal images of IF staining of cultured cells from control (top) and homozygous (bottom) tumors. The residual cells that escaped ablation and are still expressing PTHrP also express AKT1, although a small level of AKT1 is detectable in PTHrP-negative cells. Shown are DAPI (blue), PTHrP (green), and AKT1 (red). (F) Western blot of tumor extracts for AKT1 Ser473 phosphorylation. Scale bars: $50 \mu \mathrm{m}$.

ing organisms $(48,49)$. Tumor cells, including those from breast cancer, produce chemokine receptors that promote adhesion to specific cells presenting the appropriate surface ligand (the known ligand for CXCR4 is CXCL12). Organs with the highest expression of CXCL12 correlate with the most common breast cancer metastasis sites (50). The consequence is not only adhesion of the tumor cell to the ligand-expressing site, but actin polymerization and pseudopodia formation, resulting in enhanced invasiveness $(48,49)$. The CXCR4/CXCL12 combination is the most important chemokine mechanism regulating metastatic potential to the bone marrow, lungs, liver, and brain $(49,50)$. CXCR4 has been identified as a signature gene in primary breast cancers expressing the lung and the bone metastasis signature $(51,52)$; its expression in breast tumors increases with cancer progression (53) and correlates with poor survival rates (54). A study of breast cancer patients who developed skeletal metastases confirmed a generalized elevated expression for both PTHrP and CXCR4 (55).

In breast and prostate cancer cells, the CXCR4 receptor/CXCL12 ligand interaction results in activation of the PI3K/AKT pathway, which drives cell growth and survival (56) and is a key signaling route for CXCR4 $(50,57)$. The PI3K/AKT pathway is also essential to the transmission of oncogenic signaling from the middle $\mathrm{T}$ oncoprotein and to development of mammary adenocarcinomas in the PyMT system $(39,58)$. Here, Pthrp ablation and consequent
CXCR4 depletion were accompanied by a significant decrease in total levels of the prosurvival AKT1 isoform and by an increase in the AKT2 isoform. AKT2 ablation accelerates tumor induction, and the 2 AKT isoforms possess opposite oncogenic effects (59). In striking similarity with our observations, a knockout of Akt1 interferes with mammary tumor initiation and growth in PyMTMMTV mice, without hindrance to mammary development (59), but it is difficult to speculate whether PTHrP acts through acute or adaptive effect on AKT and CXCR4 signaling. Pthrp ablation has repercussions on the cell cycle $\left(\mathrm{G}_{0} / \mathrm{G}_{1}\right.$ to $\mathrm{S}$ transition, cyclin $\mathrm{D} 1$, and Ki67) and enhances apoptotic events. Similarly, in the PyMTAkt1 knockout mice, the delay in tumorigenesis is related to inhibition of cell proliferation, to a decrease in Ki67 and cyclin D1, and to promotion of apoptosis (59). Interestingly, cotargeting Pthrp and $A k t 1$ by gene ablation and siRNA techniques provides a stronger inhibition of proliferation over either blockade alone (Figure 7, $\mathrm{A}-\mathrm{B})$, suggesting the existence of PTHrP-independent signaling through AKT. This is supported by the presence of residual AKT1 in Pthrp-ablated cells (Figure 6E) and suggests that a combined therapeutic approach could provide enhanced efficacy.

The CXCR4/CXCL12 interaction drives metastatic progression by activation of angiogenesis, chemotaxis, adhesion, and invasion processes (50), and neutralization of CXCR4/CXCL12 action in vivo by antibodies (48), inhibitory peptide (60), or siRNA (61) attenuates metastases in mouse models. Here, Pthrp-targeted ablation also reduces the number of tumor cells in peripheral blood and bone marrow and delays metastasis in lungs, the preferred metastatic site in the PyMT-MMTV mouse. Importantly, the metastatic tumors that eventually develop in homozygous animals present a pattern of positive expression for both PTHrP and CXCR4, indicating that it is mainly the tumor cells that escaped Pthrp ablation that are selected to create the invasive population that develops into metastases. Since almost all residual expression of PTHrP and CXCR4 in homozygous primary tumors colocalizes to the same cells, PTHrP appears to be an important control for CXCR4 expression, with consequences to survival and metastasis. An involvement of PTH in CXCL12 production by osteoblasts (62) reinforces the concept that as a member of the PTH family, PTHrP plays a role in CXCR4/CXCL12 signaling.

Previous work with anti-PTHrP antibodies revealed inhibition of bone turnover and osteolytic metastases $(63,64)$. Here, treatment of nude mice bearing PTHrP-positive human breast cancer

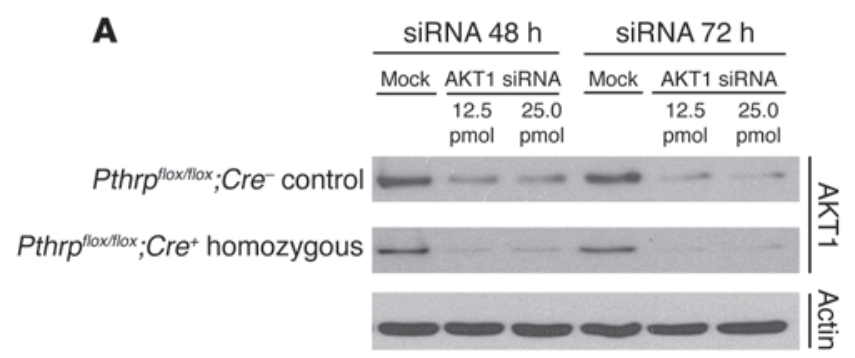

Figure 7

AKT1 inhibition by siRNA enhances Pthrp ablation effect on tumor cell growth inhibition. (A) Western blots for AKT1 expression in control (top) and homozygous cells (bottom) transfected with AKT1 siRNA or mock sequence (48 or 72 hours). (B) Proliferation of isolated Pthrpflox/flox tumor cells from control (Cre-) or homozygous ( $\mathrm{Cre}^{+}$) mice after AKT1 siRNA or mock transfection (72 hours). Representative experiment out of 3 replicates. ${ }^{* \star} P<0.05$ for all except Cre- mock versus $C^{+}{ }^{+}$siRNA: $P<0.0001$. Error bars represent SD. 
A

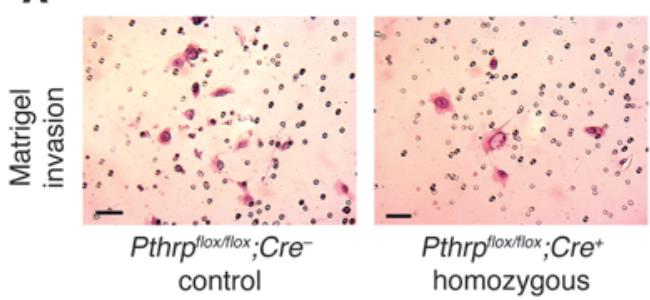

B

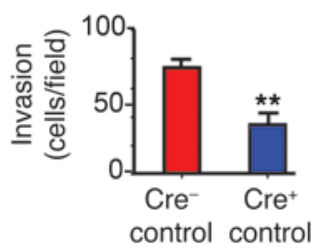

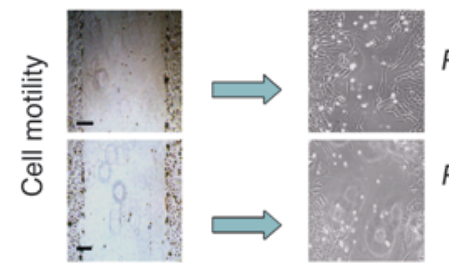

Pthrp floxfiliox;Crecontrol

Pthrp floxmilox; Cret homozygous
C

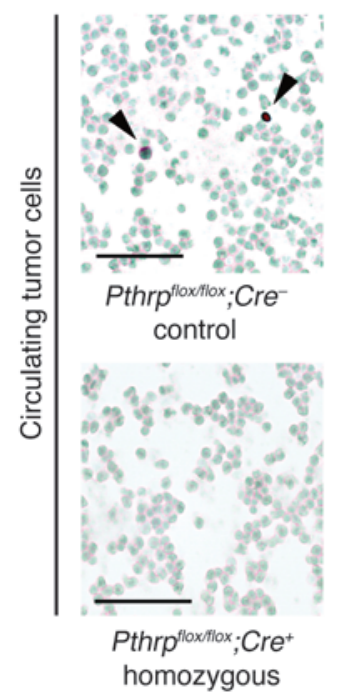

D

Tumor cells flushed from bone marrow

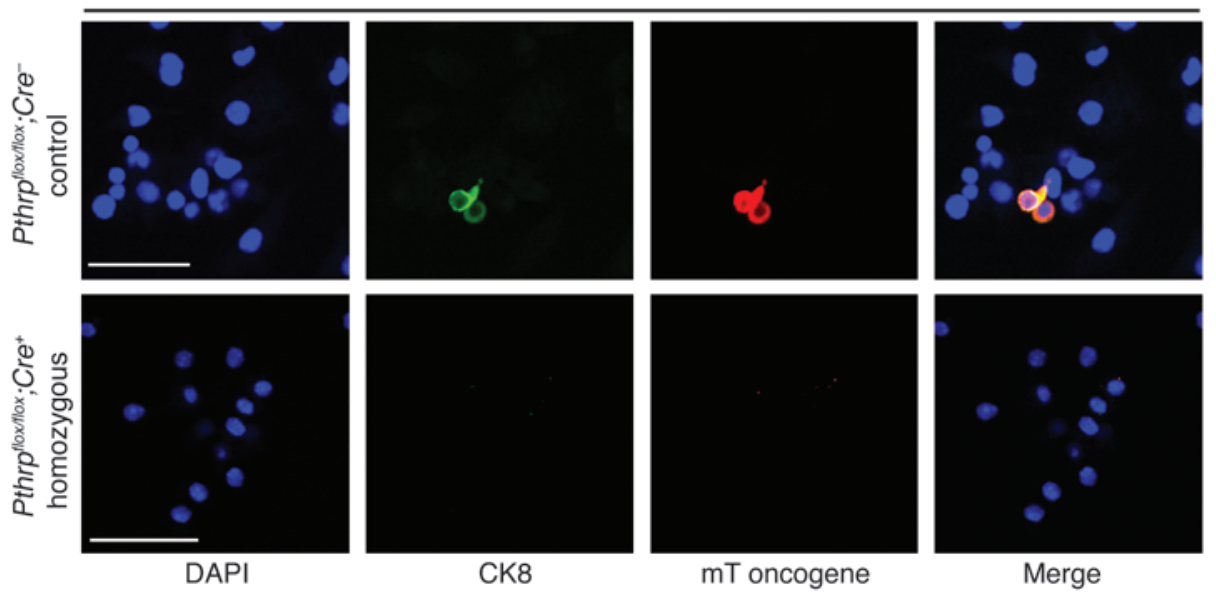

E Lung metastases (no adenovirus) $\quad \mathbf{F}$
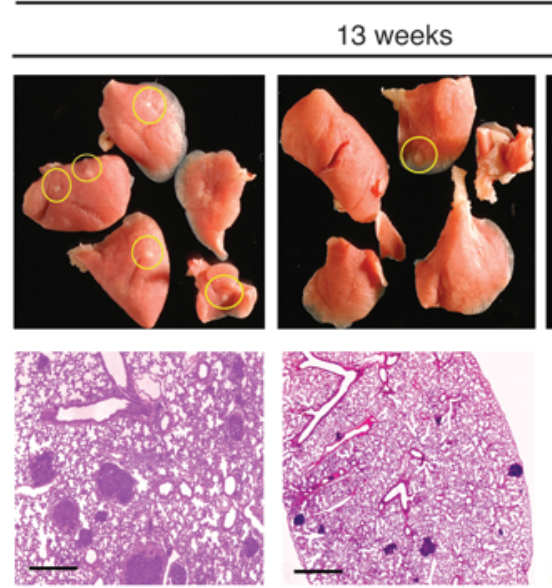

Pthrp $p^{\text {flox/lox }} ; \mathrm{Cre}^{-}$ control *

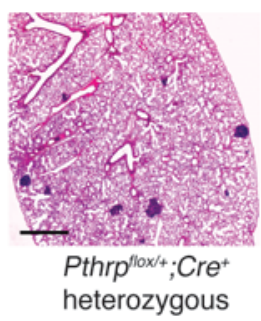

heterozygous
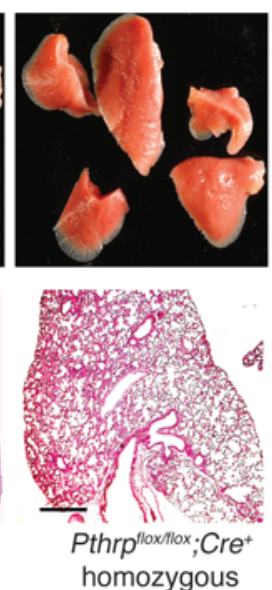

homozygous
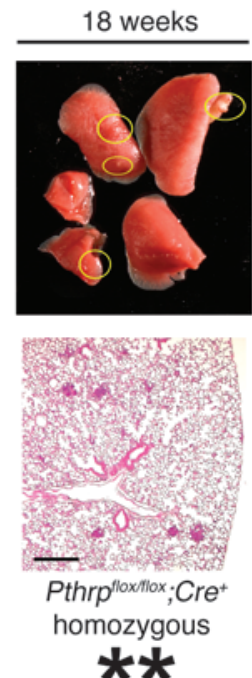

**

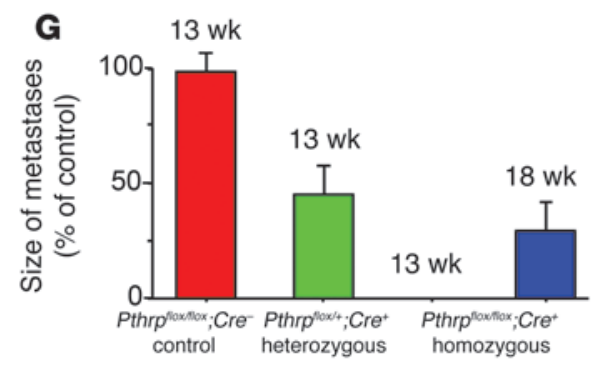

H

$\frac{\text { Lung metastases (adenovirus) }}{8 \text { weeks }}$

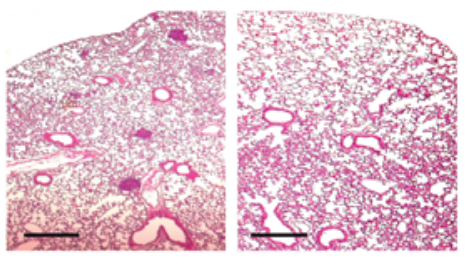

Pthrptoxtlox:

AdenoGFP

control

Pthrptloxilox:

AdenoCreGFP

homozygous

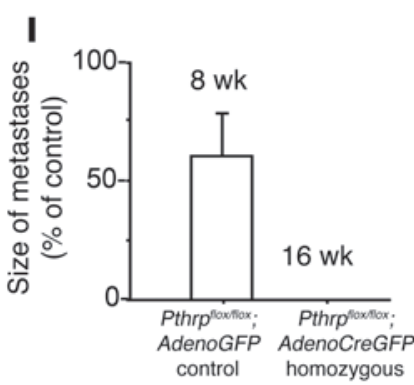




\section{Figure 8}

PTHrP drives metastatic spread. (A) Matrigel growth of tumor cells from Pthrpflox/flox; Cre $^{-}$or Pthrp flox/flox; Cre ${ }^{+}$tumors and histogram showing reduced invasive capacity for ablated cells (22 hours). ${ }^{*} P<0.01$. (B) Cell motility test after wounding (48 hours); Pthrp-ablated cells show slower motility than control cells. (C) Epimet stain of cytospins for detection of circulating tumor cells (arrowheads show pan-cytokeratin-positive cells, 18 weeks). No tumor cells are detectable in blood of homozygous mice. (D) Tumor cells flushed from bone marrow (IF stain: cytokeratin 8/PyMT double positives) in control animals only. (E) Lung metastases are slower to appear in heterozygous mice. (F and $\mathbf{G})$ Lung metastases appear in homozygous mice even later (between 13 and 18 weeks). (H and I) Lung metastases after MFP injection of adenovirus-transfected tumor cells. Lung metastases appear earlier in control mice than in the spontaneous tumor model ( $E$ and $\mathbf{G}$ ), but are not detectable in homozygous ablated mice (adenovirus transfected) at 16 weeks. (All groups for $\mathbf{E}$ to $\mathbf{I}, n=9$ ). Scale bars: $100 \mu \mathrm{m}(\mathbf{A}-\mathbf{C}) ; 50 \mu \mathrm{m}(\mathbf{D}) ; 200 \mu \mathrm{m}(\mathbf{E}, \mathbf{F}$, and $\mathbf{H})$. Error bars represent SD. Large single and double asterisks refer to corresponding stages in Figure 9.

xenografts with anti-PTHrP mAbs reduced both primary growth and lung metastatic tumor development, which suggests a PTHrP role distinct from bone turnover control and calcium homeostasis. Overall, our data demonstrate that in a model of early-onset, pregnancy-independent, highly aggressive breast tumorigenesis, PTHrP plays a tumor-promoting role in initiation, progression, and metastasis, in part through upregulation of the CXCR4 and AKT pathways (Figure 11). Autocrine, intracrine, and paracrine effects have been reported for $\operatorname{PTHrP}(8,27,65)$ and are likely involved in its pleiotropic cancer-promoting activities, since the PTH1 receptor is present throughout the tumor tissue and is unaffected by Pthrp ablation. In carcinomas, there is frequent alteration in the expression of many growth factors involved as autocrine and paracrine mediators of stromal-epithelial interactions (66). Our results support the idea that some PTHrP-mediated effects observed in vivo cannot be observed in vitro, which points to important tumor/stromal interactions that must be considered when examining PTHrP functions during breast cancer progression. Finally, the effective inhibition of PTHrP action in human cancer xenografts by our neutralizing antibodies constitutes a proof of principle for therapeutic consideration.

\section{Methods}

Conditional ablation of the Pthrp gene in mouse ME. C57BL/6 Pthrp floxed mice $($ Pthrp flox/flox) (38) were backcrossed 9 generations to FVB animals. Marker analysis (Jackson Laboratory) confirmed a more than 99\% FVB/NJ background. Crossing these mice with PyMT-MMTV strain PyMT 634 (34) or MMTV-Cre (35) mice (FVB background, Cre7 type) produced PyMT-MMTV;Pthrfflox/flox and PyMT-MMTV;Pthrfflox/+ and MMTV-Cre;Pthrpflox/flox and MMTV-Cre; Pthrfflox/+ mice. Crossing these animals produced PyMT-MMTV;Pthrpflox/flox; $\mathrm{Cre}^{+}$(homozygous), PyMT-MMTV;Pthrp flox/+; $\mathrm{Cre}^{+}$(heterozygous), PyMTMMTV; and Pthr $p^{f l o x / f l o x} ; \mathrm{Cre}^{-}$or PyMT-MMTV;Pthr $p^{f l o x /+} ; \mathrm{Cre}^{-}$(controls) mice.
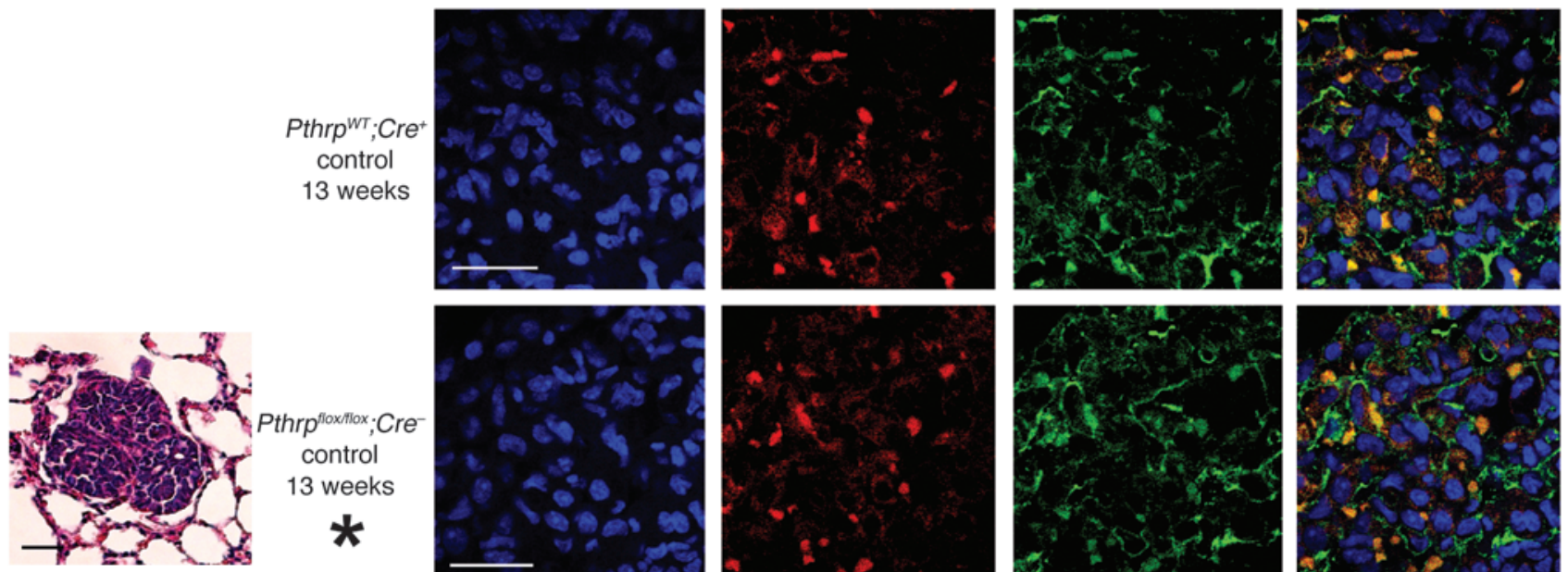

control

13 weeks
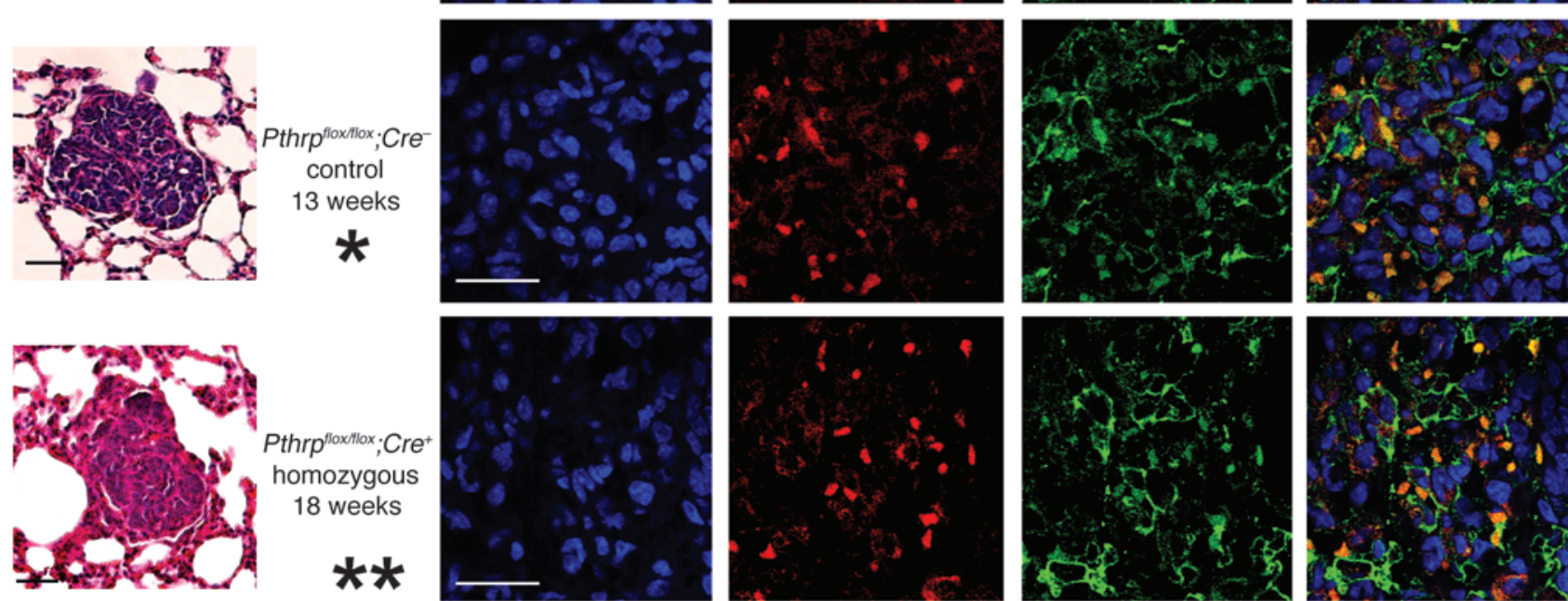

DAPI

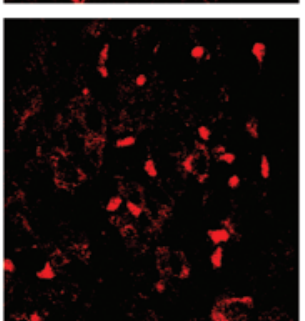

PTHrP

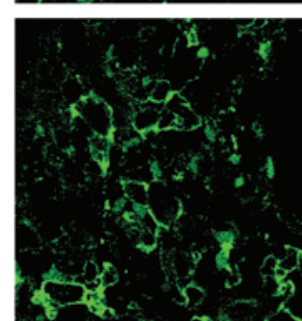

CXCR4

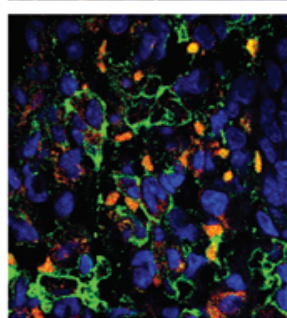

Merge

Figure 9

Spontaneous lung metastases are PTHrP and CXCR4 positive. H\&E stain (left) and IF confocal (right) of spontaneous lung metastases (no adenovirus) at same-size tumor (control 13 weeks, homozygous 18 weeks); lung metastases in homozygous mice are PTHrP and CXCR4 positive. Shown are DAPI (blue), CXCR4 (green), and PTHrP (red). Large single and double asterisks indicate corresponding stages illustrated in Figure 8E. Scale bars: $50 \mu \mathrm{m}$. 
A
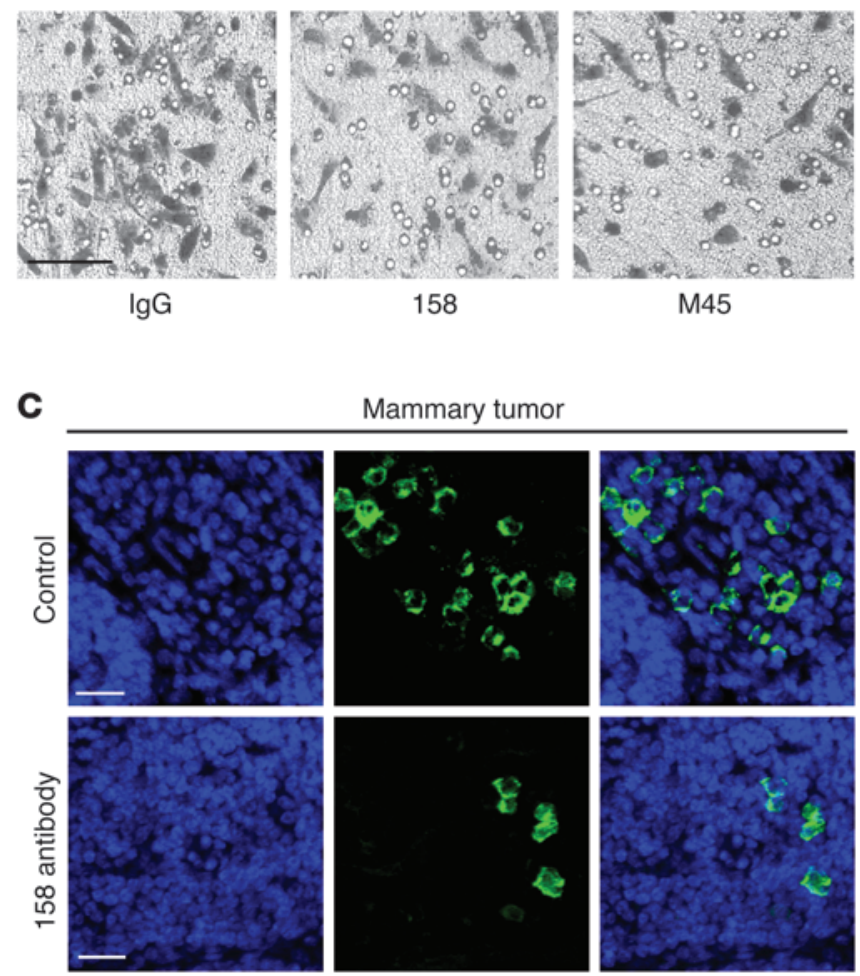

Merge
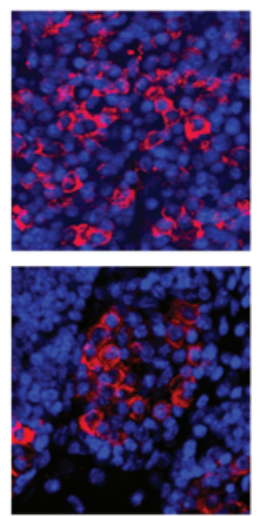

Merge
C

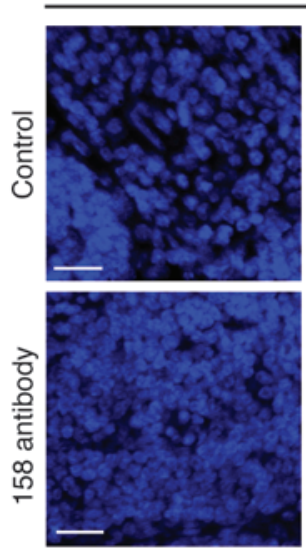

DAPI
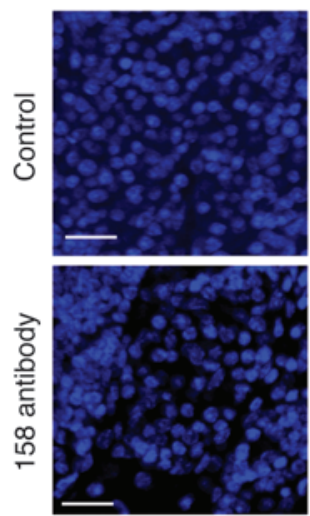

DAPI

Mammary tumor

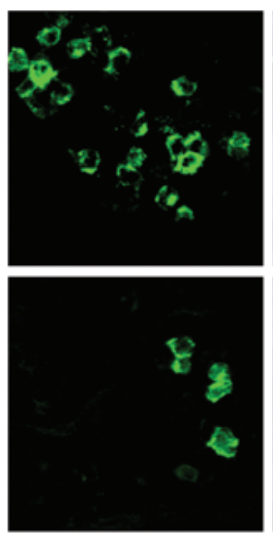

CXCR4
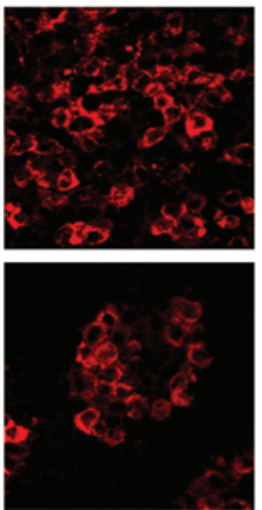

AKT1

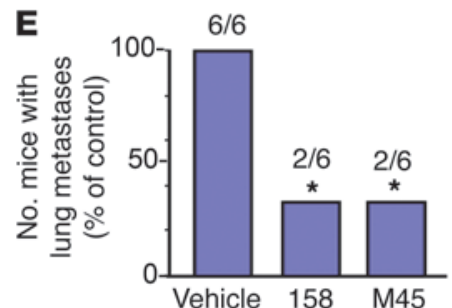

F

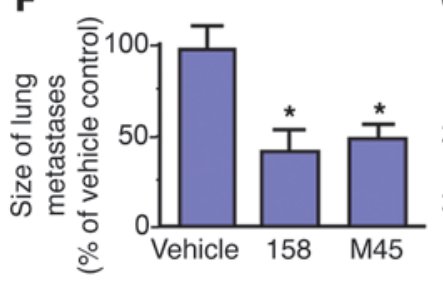

G

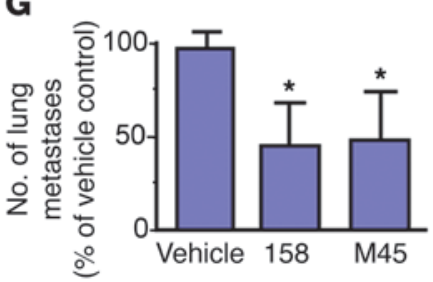

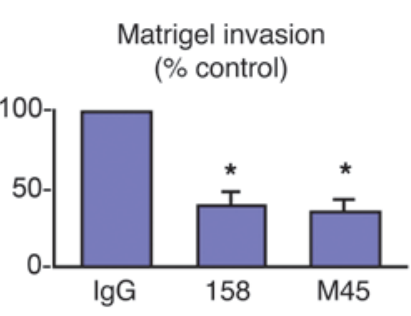

B

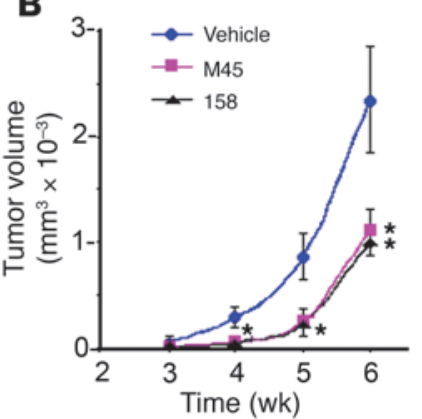

D

Lung metastases

H

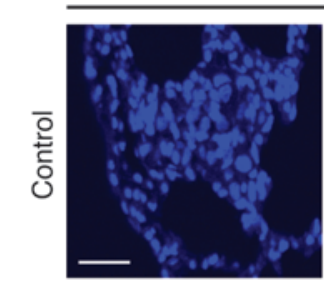

Lung metastases

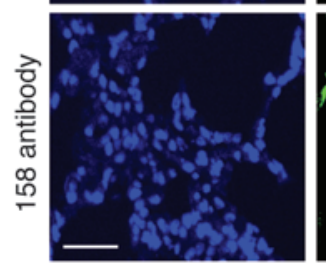

DAPI
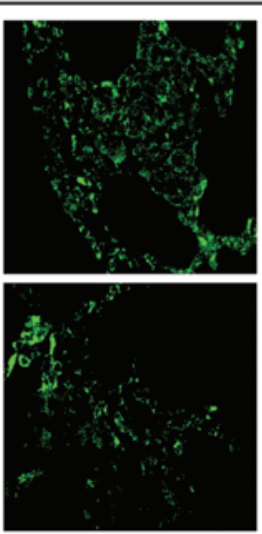

CXCR4

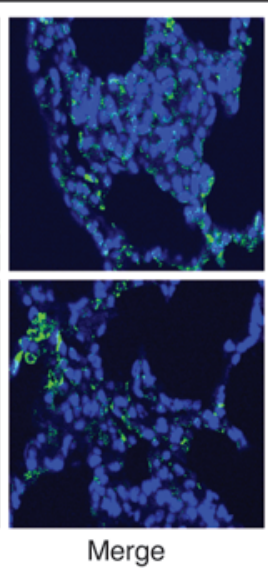




\section{Figure 10}

Anti-PTHrP neutralizing mAbs inhibit breast cancer progression in vitro and in vivo. (A) Proliferation in Matrigel (24 hours) of human MDA-MB435 breast cancer cells showing growth-inhibition effect of neutralizing antibodies 158 and M45 in vitro. ${ }^{*} P<0.05$. (B) Tumor volume over time after injection of MDA-MB-435 cells into the MFPs of BALB/c nu/nu mice and treatment with anti-PTHrP mAbs, showing the tumor-reducing effect in vivo. Data are expressed as means of 8 mice in each group. ${ }^{\star} P<0.05 ;{ }^{* *} P<0.01$. (C) IF confocal images of mammary tumors 6 weeks after injection of MDA-MB-435 cells in MFPs of nude mice showing decrease of CXCR4 (top panels) and AKT1 (bottom panels) in treated animals. Shown are DAPI (blue), CXCR4 (green), and AKT1 (red). (D) H\&E staining of lung metastases 6 weeks after injection of MDA-MB-435 in MFPs. Treatment with anti-PTHrP mAbs reduces the size and numbers of lung metastases. (E-G) Fewer mice present lung metastases after treatment with either $\mathrm{mAb}$, and the metastases are smaller and fewer in numbers in treated animals. Mean \pm SEM. ${ }^{*} P<0.05$ (E); ${ }^{*} P=0.013(\mathbf{F}) ;{ }^{*} P=0.045$ (G). (H) IF confocal images of lung metastases in nude mice injected with MDA-MB-435 cells treated ( 6 weeks) or not with anti-PTHrP mAbs. Lung metastases are CXCR4 positive irrespective of treatment. Shown are DAPI (blue) and CXCR4 (green). Scale bars: $100 \mu \mathrm{m}$ (A); $50 \mu \mathrm{m}$ (C and H); $200 \mu \mathrm{m}$ (D).

Immunohistochemistry and IF. Antibodies used were as follows: mouse monoclonal N-terminal anti-PTHrP antibody (1-34, AE-0502, IDS), mouse monoclonal PTH1R antibody (Upstate), mouse monoclonal anti-Cre recombinase and anti-phospho-Akt1 (Ser473) (Abcam), goat anti-total Akt1, Akt2, and Ki67, mouse cyclin D1, Bcl-2, actin, and rabbit polyclonal antibodies for the PTHR1 receptor, PTHrP ${ }_{41-139}$ C-terminal end, tubulin, anti-rabbit and anti-mouse peroxidase conjugates (Santa Cruz Biotechnology Inc.), Alexa Fluor 555-conjugated goat anti-rabbit IgG and Alexa Fluor 488-conjugated goat anti-mouse IgG (Invitrogen), guinea pig anti-mouse cytokeratin 8, donkey anti-guinea pig IgG CY3-labeled, and rabbit anti-mouse cytokeratin 5 (PROGEN Biotechnik GmbH).

Tumors and other tissues were fixed, embedded, sliced, and stained with $\mathrm{H} \& \mathrm{E}$ according to standard protocols. The SK-4100 kit (Victor) was used for immunohistochemistry (IHC), and slides were reacted for diaminobenzidine and analyzed with a Leica DMR microscope and BIOQUANT Nova Prime software (Bioquant). IF staining was conducted on deparaffinized tissue sections or cells fixed on slides. Results were analyzed with an LSM 510 Meta confocal microscope (Carl Zeiss Microimaging).

Whole-mount staining. MFPs were removed, spread on a glass slide, air dried, fixed, delipidated, and stained with $0.5 \%$ Neutral Red (Sigma-Aldrich).

Western blotting. Proteins were extracted from tissues, and samples $(30-50 \mu \mathrm{g})$ were fractionated by SDS-PAGE electrophoresis, transferred to PVDF membranes, reacted with primary and secondary antibodies, and developed by enhanced chemiluminescence according to standard methods. Whole mammary glands were prepared according to Ackler et al. (67).

Culture of mouse breast tumor cells from primary tumors. Tumors were harvested, minced, and incubated in $2.4 \mathrm{mg} / \mathrm{ml}$ collagenase $\mathrm{B}$ and dispase II (Roche) in DMEM (no FBS) at $37^{\circ} \mathrm{C}$ for 2 hours. Floating cells were washed, pelleted, resuspended, and propagated in complete DMEM.

Adenovirus vector. Tumor cells in culture were infected with a recombinant adenovirus vector $\left(10^{8}\right.$ viral particles $\left./ \mathrm{ml}\right)$ containing a GFP-Cre transgene for the Cre recombinase.
Forty-eight hours later, cells were selected on a BD FACSAria II cell sorter. GFP-positive cells $\left(1 \times 10^{6}\right.$ cells $)$ were injected into the fourth MFP of anesthetized 5-week-old syngeneic FVB mice (Charles River).

Cell-cycle analysis, PTHrP, and calcium assays. Cultured cells were stained with propidium iodide and analyzed for cell cycle by flow cytometry (FACSCalibur). Levels of PTHrP in culture medium or serum were measured by 2 -site immunoradiometric assay (Beckman-Coulter). Serum calcium was determined with a Synchron 67 Autoanalyzer (Beckman).

siRNA transfection for Akt1 knockdown. Cells isolated from mammary tumors were transfected with siRNA Akt1 targeting sequences (s62216) as follows: sense: CUCAAGUACUCAUUCCAGAtt, antisense: UCUGGAAUGAGUACUUGAGgg (bases in lower case are overhangs not corresponding to the original target sequence), and negative control (Ambion). Cell proliferation was assayed by hemocytometer counting.

Tumor cell detection in peripheral blood and in bone marrow. Blood was collected from sacrificed animals by cardiac puncture. Cytospin-concentrated samples were stained with the Epimet Epithelial Cell Kit (Micromet). Bone marrow flushed with PBS from tibias and femurs cut at the knee joint was tritrated through a 25 -gauge needle. Samples (2 tibias and 2 femurs/ animal) were counted with a hemocytometer and spread onto Cytospin Immunoselect Adhesion Slides (Squarix). Tumor cells were detected with pan-cytokeratin mAb A45-B/B3 or cultured in DMEM with 10\% FBS.

Matrigel invasion and wound-healing assays. MDA-MB-435 cells were tested for invasion in Matrigel for 20-24 hours with or without mAbs or IgG $(10 \mu \mathrm{g} / \mathrm{ml})$ according to standard methods. Invading cells were counted after paraformaldehyde fixation and hematoxylin staining. For motility assays, cells were grown on poly-D-lysine-coated plates (Millipore). The cell carpet was scraped and the growth of cells into the scraped area monitored.

Lung metastases measurements. Photomicrographs of H\&E-stained slides of lung metastases were analyzed with ImageJ software (http://rsbweb.nih. gov/ij/index.html) to assay metastatic surface/lung ( $n=9$ mice).

Anti-PTHrP(1-33) $m A$ ss. We generated 2 PTHrP-specific mAbs against $\mathrm{hPTHrP}_{1-33}$ peptide; hybridomas 158 and M45 produced mAbs (sub-

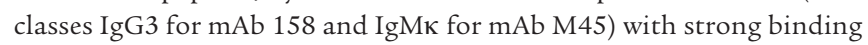
to hPTHrP ${ }_{1-33}$. The mAbs were highly specific (no reaction with PTH), and no cross-reactivity between antibodies and other fragments of PTHrP was observed. The 158 and M45 hybridomas have been deposited at the International Depositary Authority of Canada (accession numbers 060808-02 and 060808-01).

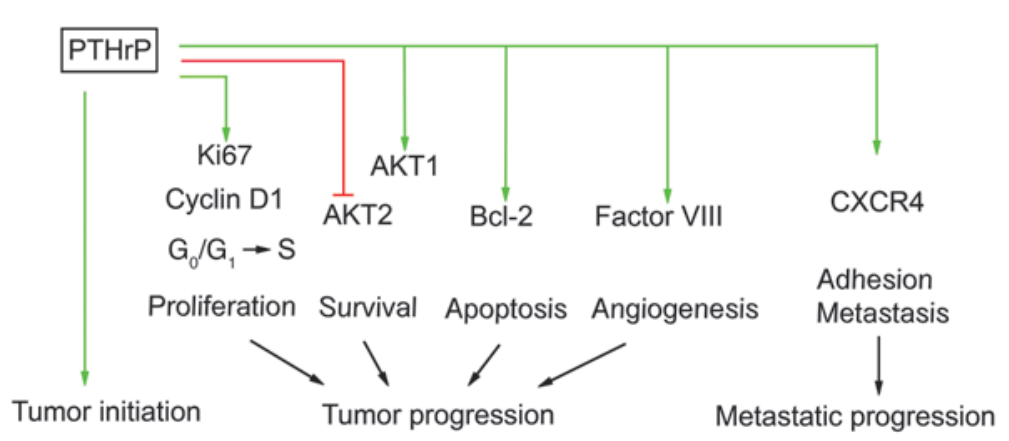

Figure 11

PTHrP influences several key steps in breast cancer. Interactions are described here for $\mathrm{PTHrP}$ in tumor cell proliferation; through its effects on cell proliferation factor Ki67, cell-cycle progression regulator cyclin $\mathrm{D} 1$, and the $\mathrm{G}_{0} / \mathrm{G}_{1}$ to $\mathrm{S}$ transition, PTHrP is involved in very early steps of oncogenesis. PTHrP influences breast tumor cell survival, apoptosis, and angiogenesis through control of levels of expression for crucial signaling molecules such as AKT1/AKT2, Bcl-2, and factor VIII. Of great interest is the observation that PTHrP is involved in the control of CXCR4 expression and consequently also plays a role in metastatic spread. 
Treatment of nude mice with anti-PTHrP $m A$ Abs. $1 \times 10^{6}$ human cancer cells (MDA-MB-435) were injected into the fourth MFP of 4- to 5-week-old $\mathrm{BALB} / \mathrm{C} \mathrm{nu} / \mathrm{nu}$ females (Charles River). Starting the next day, antibodies were injected subcutaneously $(200 \mu \mathrm{g})$ every 48 hours for 6 weeks. Mouse $\mathrm{IgG}_{3}$ isotype control was from R\&D Systems. Primary tumor growth rates were determined by plotting the means of 2 orthogonal diameters of the tumor measured at 5-day intervals. Tumor volume was measured once weekly. Animals were sacrificed 6 weeks after tumor implantation.

PyMT-MMTV animal handling. Tumor-bearing animals were examined by palpation twice a week until they were 13 weeks of age. Mice with excessive tumor burden $(>1.5 \mathrm{~cm})$ were euthanized. For Kaplan-Meier, animals were sacrificed when total tumor load exceeded $20 \%$ of animal weight. All experiments were carried out in compliance with regulations of the McGill University Institutional Animal Care Committee. All animal surgeries were conducted in accordance with principles and procedures dictated by the highest standards of humane animal care.

Statistical analysis of tumor progression and tumor growth. Numerical data are presented as the mean \pm SD. The data were analyzed by ANOVA followed by a Bonferroni's post-test to determine the statistical significance of differences. All statistical analyses were performed using Instat Software
(GraphPad Software), and $P<0.05$ was considered statistically significant. Specific tests are mentioned in the text.

Study approval. These animal studies were approved by the McGill University Animal Compliance Office.

\section{Acknowledgments}

This work was supported by Canadian Institutes for Health Research (CIHR) grants MOP 10839 (to R. Kremer) and MOP 102555 (to R. Kremer and A. Karaplis); by Susan G. Komen Foundation grant KG 100766 (to R. Kremer and A. Karaplis); and by US Department of Defense BCRP Concept Award BC023897 (to A. Karaplis). We thank Jing Lian and Bill Papavasiliou for technical help.

Received for publication December 17, 2010, and accepted in revised form September 21, 2011.

Address correspondence to: Richard Kremer, Department of Medicine, McGill University Health Centre, 687 Pine Ave. West, Montréal, Quebec, Canada, H3A 1A1. Phone: 514.843.1632; Fax: 514.843.1712; E-mail: richard.kremer@mcgill.ca.
1. Mundy GR. Metastasis: Metastasis to bone: causes, consequences and therapeutic opportunities. Nat Rev Cancer. 2002;2(8):584-593.

2. Roodman GD. Mechanisms of bone metastasis. NEngl J Med. 2004;350(16):1655-1664.

3. Di Cosimo S. Controversies in breast cancer: the mammalian target of rapamycin as a target for breast cancer therapy. Breast Cancer Res. 2009; 11(suppl 3):S25.

4. Tabernero J, et al. Dose- and schedule-dependent inhibition of the mammalian target of rapamycin pathway with everolimus: a phase I tumor pharmacodynamic study in patients with advanced solid tumors. J Clin Oncol. 2008;26(10):1603-1610.

5. Gensure RC, Gardella TJ, Juppner H. Parathyroid hormone and parathyroid hormone-related peptide, and their receptors. Biochem Biophys Res Commun. 2005;328(3):666-678.

6. Maioli E, Fortino V. The complexity of parathyroid hormone-related protein signalling. Cell Mol Life Sci. 2004;61(3):257-262.

7. Miao D, et al. Severe growth retardation and early lethality in mice lacking the nuclear localization sequence and C-terminus of PTH-related protein. Proc Natl Acad Sci U S A. 2008;105(51):20309-20314.

8. Philbrick WM, Dreyer BE, Nakchbandi IA, Karaplis AC. Parathyroid hormone-related protein is required for tooth eruption. Proc Natl Acad Sci U S A. 1998;95(20):11846-11851.

9. Karaplis AC, et al. Lethal skeletal dysplasia from targeted disruption of the parathyroid hormonerelated peptide gene. Genes Dev. 1994;8(3):277-289.

10. Wysolmerski JJ, Philbrick WM, Dunbar ME, Lanske B, Kronenberg H, Broadus AE. Rescue of the parathyroid hormone-related protein knockout mouse demonstrates that parathyroid hormone-related protein is essential for mammary gland development. Development. 1998;125(7):1285-1294.

11. Bucht E, et al. Parathyroid hormone-related protein in patients with primary breast cancer and eucalcemia. Cancer Res. 1998;58(18):4113-4116.

12. Kohno N, et al. The expression of parathyroid hormone-related protein in human breast cancer with skeletal metastases. Surg Today. 1994;24(3):215-220.

13. Brandt DW, Burton DW, Gazdar AF, Oie HE, Deftos LJ. All major lung cancer cell types produce parathyroid hormone-like protein: heterogeneity assessed by high performance liquid chromatography. Endocrinology. 1991;129(5):2466-2470.

14. Kitazawa S, et al. Immunohistologic evaluation of parathyroid hormone-related protein in human lung cancer and normal tissue with newly developed monoclonal antibody. Cancer. 1991; 67(4):984-989.

15. Nishigaki Y, Ohsaki Y, Toyoshima E, Kikuchi K. Increased serum and urinary levels of a parathyroid hormone-related protein $\mathrm{COOH}$ terminus in non-small cell lung cancer patients. Clin Cancer Res. 1999;5(6):1473-1481.

16. Dougherty KM, et al. Parathyroid hormone-related protein as a growth regulator of prostate carcinoma. Cancer Res. 1999;59(23):6015-6022.

17. Iwamura $M$, et al. Immunohistochemical localization of parathyroid hormone-related protein in human prostate cancer. Cancer Res. 1993; 53(8):1724-1726.

18. Kremer R, Goltzman D, Amizuka N, Webber MM, $\mathrm{J} / \mathrm{S} / \mathrm{R}$. ras Activation of human prostate epithelial cells induces overexpression of parathyroid hormone-related peptide. Clin Cancer Res. 1997; 3(6):855-859.

19. Agouni A, et al. Parathyroid hormone-related protein induces cell survival in human renal cell carcinoma through the PI3K Akt pathway: evidence for a critical role for integrin-linked kinase and nuclear factor kappa B. Carcinogenesis. 2007;28(9):1893-1901.

20. Nishihara $M$, Ito $M$, Tomioka $T$, Ohtsuru $A$, Taguchi T, Kanematsu T. Clinicopathological implications of parathyroid hormone-related protein in human colorectal tumours. J Pathol. 1999; 187(2):217-222.

21. Nishihara M, Kanematsu T, Taguchi T, Razzaque MS. PTHrP and tumorigenesis: is there a role in prognosis? Ann N Y Acad Sci. 2007;1117:385-392.

22. Dihlmann S, Kloor M, Fallsehr C, von Knebel Doeberitz M. Regulation of AKT1 expression by betacatenin/Tcf/Lef signaling in colorectal cancer cells. Carcinogenesis. 2005;26(9):1503-1512.

23. El Abdaimi K, Papavasiliou V, Goltzman D, Kremer R. Expression and regulation of parathyroid hormone-related peptide in normal and malignant melanocytes. Am J Physiol Cell Physiol. 2000; 279(4):C1230-C1238.

24. Henderson J, Sebag M, Rhim J, Goltzman D, Kremer R. Dysregulation of parathyroid hormone-like peptide expression and secretion in a keratinocyte model of tumor progression. Cancer Res. 1991; 51(24):6521-6528.

25. Alipov GK, et al. Expression of parathyroid hormone-related peptide (PTHrP) in gastric tumours. J Pathol. 1997;182(2):174-179.

26. Han Z, et al. Akt1/protein kinase B alpha is involved in gastric cancer progression and cell proliferation. Dig Dis Sci. 2008;53(7):1801-1810.

27. Deftos LJ, Barken I, Burton DW, Hoffman RM, Geller J. Direct evidence that PTHrP expression promotes prostate cancer progression in bone. Biochem Biophys Res Commun. 2005;327(2):468-472.

28. Firkin F, Seymour JF, Watson AM, Grill V, Martin TJ. Parathyroid hormone-related protein in hypercalcaemia associated with haematological malignancy. Br J Haematol. 1996;94(3):486-492.

29. Kissin MW, Henderson MA, Danks JA, Hayman JA, Bennett RC, Martin TJ. Parathyroid hormone related protein in breast cancers of widely varying prognosis. Eur J Surg Oncol. 1993;19(2):134-142.

30. Kremer R, Shustik C, Tabak T, Papavasiliou V, Goltzman D. Parathyroid-hormone-related peptide in hematologic malignancies. Am J Med. 1996; 100(4):406-411.

31. Rankin W, Grill V, Martin TJ. Parathyroid hormone-related protein and hypercalcemia. Cancer. 1997;80(8 suppl):1564-1571.

32. Truong NU, deB Edwardes MD, Papavasiliou V, Goltzman D, Kremer R. Parathyroid hormone-related peptide and survival of patients with cancer and hypercalcemia. Am J Med. 2003;115(2):115-121.

33. Dittmer A, et al. Parathyroid hormone-related protein regulates tumor-relevant genes in breast cancer cells. J Biol Chem. 2006;281(21):14563-14572.

34. Guy CT, Cardiff RD, Muller WJ. Induction of mammary tumors by expression of polyomavirus middle $\mathrm{T}$ oncogene: a transgenic mouse model for metastatic disease. Mol Cell Biol. 1992;12(3):954-961.

35. Andrechek ER, Hardy WR, Siegel PM, Rudnicki MA, Cardiff RD, Muller WJ. Amplification of the neu/erbB-2 oncogene in a mouse model of mammary tumorigenesis. Proc Natl Acad Sci U S A. 2000; 97(7):3444-3449.

36. Wysolmerski JJ, McCaughern-Carucci JF, Daifotis AG, Broadus AE, Philbrick WM. Overexpression of parathyroid hormone-related protein or parathyroid hormone in transgenic mice impairs branching morphogenesis during mammary gland development. Development. 1995;121(11):3539-3547.

37. Wagner KU, McAllister K, Ward T, Davis B, Wiseman R, Hennighausen L. Spatial and temporal expression of the Cre gene under the control of the MMTV-LTR in different lines of transgenic mice. Transgenic Res. 2001;10(6):545-553.

38. He B, et al. Tissue-specific targeting of the pthrp gene: the generation of mice with floxed alleles. Endocrinology. 2001;142(5):2070-2077. 
39. Lin EY, et al. Progression to malignancy in the polyoma middle $\mathrm{T}$ oncoprotein mouse breast cancer model provides a reliable model for human diseases. Am J Pathol. 2003;163(5):2113-2126.

40. Hiraki A, et al. Parathyroid hormone-related protein measured at the time of first visit is an indicator of bone metastases and survival in lung carcinoma patients with hypercalcemia. Cancer. 2002; 95(8):1706-1713

41. Liao J, et al. Tumor expressed PTHrP facilitates prostate cancer-induced osteoblastic lesions. Int J Cancer. 2008;123(10):2267-2278.

42. Linforth R, et al. Coexpression of parathyroid hormone related protein and its receptor in early breast cancer predicts poor patient survival. Clin Cancer Res. 2002;8(10):3172-3177.

43. Pecherstorfer $M$, et al. Parathyroid hormonerelated protein and life expectancy in hypercalcemic cancer patients. J Clin Endocrinol Metab. 1994; 78(5):1268-1270

44. Fleming NI, et al. Parathyroid hormone related protein protects against mammary tumor emergence and is associated with monocyte infiltration in ductal carcinoma in situ. Cancer Res. 2009; 69(18):7473-7479.

45. Hager JH, Hodgson JG, Fridlyand J, Hariono S, Gray JW, Hanahan D. Oncogene expression and genetic background influence the frequency of DNA copy number abnormalities in mouse pancreatic islet cell carcinomas. Cancer Res. 2004; 64(7):2406-2410.

46. Lifsted $\mathrm{T}$, et al. Identification of inbred mouse strains harboring genetic modifiers of mammary tumor age of onset and metastatic progression. Int J Cancer. 1998;77(4):640-644.
47. Henderson MA, et al. Parathyroid hormone-related protein production by breast cancers, improved survival, and reduced bone metastases. J Natl Cancer Inst. 2001;93(3):234-237.

48. Muller A, et al. Involvement of chemokine receptors in breast cancer metastasis. Nature. 2001; 410(6824):50-56.

49. Zlotnik A. New insights on the role of CXCR4 in cancer metastasis. J Pathol. 2008;215(3):211-213.

50. Luker KE, Luker GD. Functions of CXCL12 and CXCR4 in breast cancer. Cancer Lett. 2006; 238(1):30-41

51. Kang Y, et al. A multigenic program mediating breast cancer metastasis to bone. Cancer Cell. 2003; 3(6):537-549.

52. Minn AJ, et al. Genes that mediate breast cancer metastasis to lung. Nature. 2005;436(7050):518-524.

53. Allinen $\mathrm{M}$, et al. Molecular characterization of the tumor microenvironment in breast cancer. Cancer Cell. 2004;6(1):17-32.

54. Li YM, et al. Upregulation of CXCR4 is essential for HER2-mediated tumor metastasis. Cancer Cell. 2004;6(5):459-469.

55 . Bohn OL, et al. Biomarker profile in breast carcinomas presenting with bone metastasis. Int J Clin Exp Pathol. 2010;3(2):139-146.

56. Hennessy BT, Smith DL, Ram PT, Lu Y, Mills GB. Exploiting the PI3K/AKT pathway for cancer drug discovery. Nat Rev Drug Discov. 2005;4(12):988-1004.

57. Chinni SR, et al. CXCL12/CXCR4 signaling activates Akt-1 and MMP-9 expression in prostate cancer cells: The role of bone microenvironmentassociated CXCL12. Prostate. 2006;66(1):32-48.

58. Fluck MM, Schaffhausen BS. Lessons in signaling and tumorigenesis from polyomavirus middle $\mathrm{T}$ antigen. Microbiol Mol Biol Rev. 2009;73(3):542-563.

59. Maroulakou IG, Oemler W, Naber SP, Tsichlis PN. Akt1 ablation inhibits, whereas Akt2 ablation accelerates, the development of mammary adenocarcinomas in mouse mammary tumor virus (MMTV)-ErbB2/neu and MMTV-polyoma middle T transgenic mice. Cancer Res. 2007;67(1):167-177.

60. Hatse S, Princen K, Bridger G, De Clercq E, Schols D. Chemokine receptor inhibition by AMD3100 is strictly confined to CXCR4. FEBS Lett. 2002;527(1-3):255-262.

61. Liang Z, Yoon Y, Votaw J, Goodman MM, Williams L, Shim H. Silencing of CXCR4 blocks breast cancer metastasis. Cancer Res. 2005;65(3):967-971.

62. Jung Y, et al. Regulation of SDF-1 (CXCL12) production by osteoblasts; a possible mechanism for stem cell homing. Bone. 2006;38(4):497-508.

63. Guise TA, et al. Evidence for a causal role of parathyroid hormone-related protein in the pathogenesis of human breast cancer-mediated osteolysis. J Clin Invest. 1996;98(7):1544-1549.

64. Saito H, Tsunenari T, Onuma E, Sato K, Ogata E, Yamada-Okabe H. Humanized monoclonal antibody against parathyroid hormone-related protein suppresses osteolytic bone metastasis of human breast cancer cells derived from MDA-MB-231. Anticancer Res. 2005;25(6B):3817-3823.

65. Karaplis AC. PTHrP: novel roles in skeletal biology. Curr Pharm Des. 2001;7(8):655-670.

66. Bhowmick NA, Moses HL. Tumor-stroma interactions. Curr Opin Genet Dev. 2005;15(1):97-101.

67. Ackler S, Ahmad S, Tobias C, Johnson MD, Glazer RI. Delayed mammary gland involution in MMTV-AKT1 transgenic mice. Oncogene. 2002; 21(2):198-206. 